\title{
Epileptic-Seizure onset detection using \\ PARAFAC model with Cross-Wavelet Transformation on Multi-Channel EEG
}

\section{Arijit Ghosh}

Jadavpur University

\section{Purbanka Pahari}

Jadavpur University

Piyali Basak

Jadavpur University

Anasua Sarkar ( $\square$ ashru2006@hotmail.com )

Jadavpur University Faculty of Engineering and Technology https://orcid.org/0000-0001-7365-3924

\section{Research Article}

Keywords: Multi-channel EEG, onset seizure, wavelet cross spectrum, wavelet coherence, PARAFAC.

Posted Date: February 23rd, 2021

DOI: https://doi.org/10.21203/rs.3.rs-248320/v1

License: @ (i) This work is licensed under a Creative Commons Attribution 4.0 International License.

Read Full License 
Epileptic-Seizure onset detection using PARAFAC model with Cross-Wavelet Transformation on multi-channel EEG

\begin{abstract}
Background: Finding components from multi-channel EEG signal for localizing and detection of onset of seizure is a new approach in biomedical signal analysis. Tensor-based approaches are utilized to fit the components into multi-dimensional array in recent works.
\end{abstract}

Method: We initially decompose EEG signals into Beta band using Discrete Wavelet Transform. We compare patient templates with normal template for cross-wavelet analysis to obtain Wavelet cross spectrum and Wavelet cross coherence coefficients. Next we apply PARAFAC (Parallel Factorization) modeling, a three-way tensor-based representation in channel, frequency and time-points dimensions on features. Finally, we utilize ensemble classifier for detecting seizure-free, onset and seizure classes.

Results: The clinical dataset for this work comprises of 5 normal subjects and 6 epileptiform patients. The classification performances of Wavelet cross spectrum features on PARAFAC model for Seizure detection using Ensemble Bagged-Trees classifier obtains highest $82.21 \%$ accuracy, while for Wavelet Coherence features it provides $84.76 \%$ accuracy. The results have been compared with well-known Fine Gaussian SVM, Weighted KNN and Ensemble Subspace KNN classifiers.

Conclusions: The aim is to analyze data over three dimensions i.e., time, frequency and space (channels). This EEG based analysis is effective as an automatic method for detection of seizure before its actual manifestation.

Key words - Multi-channel EEG, onset seizure, wavelet cross spectrum, wavelet coherence, PARAFAC.

\title{
1. Introduction
}

Epileptic seizure is commonly defined by International League Against Epilepsy (ILAE) and the International Bureau for Epilepsy (IBE) as transient occurrence of signs and symptoms due to excessive abnormal or synchronous neuronal activity in the brain [1]. It occurs mainly when a burst of electrical impulses abnormally crosses their normal amplitude limits. Further it spreads to the adjoining regions of the brain, where it creates a storm of electrical activities which gets physical outcome as seizure. Seizures can be classified based on their intensities, frequency, duration. However, the pattern differs from one patient to the another, but the same pattern is repeated for the given patient for any event of the seizure. In 1981, ILAE, proposed the scheme for classification of epileptic seizure. Classification of seizure types helps the physician in diagnosing whether the patient is epileptic or not and to choose the proper tool for treatment. Based on the type of behavior and activity of brain, seizure can be divided into 2 broad classes i.e., Partial (also called Focal or Local) seizures and Generalized seizure (also called convulsive or non-convulsive). Epilepsy is a clinical syndrome resulting from various disease conditions rather than a single disease [2]. For more than $20 \%$ of the overall global epilepsy patients, neither 
medication nor respective surgery result in elimination of seizures [3]. In fact, epilepsy is marked by presence of at least one epileptic seizures [4].

Electroencephalography refers to the measurement of the electrical activities of the brain by amplification of the potential differences existing between the recording electrodes placed on scalp or on the cerebral cortex. EEG particularly manifests different types of rhythms based on the activities of the brain, specific behavioral states (sleep, wakefulness) and physio-pathological conditions (like some chronic disease or coma state) and these rhythms (delta, theta, alpha, beta) are particularly manifested in a unique frequency band. EEG having high temporal resolution, can be sensitive with the events taking place within milliseconds. It is a premium choice of diagnostic tool used for detection of seizures and presence of brain tumors. EEG signal is abnormal if it contains epileptiform activities, slow waves and even abnormalities of amplitude. However, this pattern may resemble a normal activity which even gets more cumbersome to isolate the normal signal from the abnormal EEG activity [5]. In broad sense the epochs of EEG time series with seizure activity are called ictal EEG, the previous part of the ictal EEG is called the pre-ictal EEG and the segments between two consecutive ictal episodes are termed as interictal EEG.

Here, we use EEG signals acquired from epileptic patients and normal subjects and try to develop a method for detection of seizure. Our approach starts with decomposition of EEG signal into Beta band using Discrete Wavelet Transform. After calculating Wavelet cross spectrum and Wavelet cross coherence coefficients, we use three-way canonical decomposition model, particularly by using Parallel Factor (PARAFAC) analysis to decompose into time-frequency space atoms. Further we use four classifiers to classify the time atom features into seizure-free, onset and seizure classes.

\section{Literature Review}

Early detection and prediction of epileptic seizures are of prime clinical importance. Unattended seizures can not only lead to serious physical injuries and status epilepticus, but also, they may risk in unexpected deaths. Accordingly, several studies and techniques are being evolved to detect any early sign of the seizure episodes. The studies mainly focus on extracting acquiring the raw signal and thereby extract feature parameters which gives key distinguishing characteristics in identifying an abnormal activity in the brain.

Robust seizure detection algorithms are also implemented using EEG analysis methods. Initially seizure detection algorithms dates to 1982, when Gotman designed an automated seizure detection algorithm [6]. The experiment facilitated the study of correlation of epileptic seizures and manifestation by decomposing the EEG into elementary waves and detection of paroxysmal bursts of rhythmic activities. However, their study was reluctant about the false detection rates.

Further development in automated detection of epileptic seizures has led to the study of unique patient specific technique of seizure onset detection as done by $\mathrm{Qu}$ and Gotman [7]. In their study, time and frequency domain features were extracted which were used as an input to a classifier. The system was able to detect onset of seizure with $100 \%$ accuracy.

With recent developments in detection of seizure, comparative studies were done on EEG features to design an effective algorithm, Greene et al. [8] studied a test set of 21 neonatal seizures and compared the extracted EEG features to potentially identify the seizures. Their method yielded a sensitivity of $81.08 \%$ and specificity of $82.23 \%$.

Acharya in 2013 [9], employed discrete wavelet transform based methods and independent component analysis for reducing the data dimension and effectively extract features which were then fed to 
classifiers to develop an automated classifier for diagnosing epileptic seizures. The proposed techniques had high accuracy and sensitivity for even short data duration.

Automated seizure detection using wavelet-based methods are also implemented for application to seizure detection. Xie and Krishnan in 2013, proposed one such approach using wavelet based sparse functional linear model as an input to classifier which yielded high classification results [10]. Their proposed scheme leads to $99 \%$ accuracy for the database used in their work.

Chen in 2014 proposed another wavelet-based seizure detection method by implementing dual tree complex wavelet and Fourier features [11]. The features were classified to develop the algorithm. The proposed algorithm achieved $100 \%$ classification rates.

Implementation of nonlinear adaptive models for automated detection of epilepsy has been shown in few studies. One of such studies done by Martis et al. in 2012, used empirical mode decomposition of EEG signals for yielding few intrinsic mode functions which are again transformed to get generic features for classification [12]. The evaluated performance showed an accuracy of $95.33 \%$ with sensitivity of $98 \%$ and average specificity of $97 \%$.

\section{Materials}

The clinical dataset for the present work was acquired form Long term EEG monitoring Lab at AMRI Hospital, Kolkata, India. The EEG was acquired using Nicoletc64 LTM System manufactured by Natus Neurology Inc., USA (formerly known as CareFusion Healthcare).

For this work $250 \mathrm{~Hz}$ sampling rate was selected. 21 unipolar channels (FP1, FP2, F3, F4, C3, C4, P3, P4, 01, 02, F7, F8, T3, T5, T6, Fz, Cz, Pz, A1, A2) of 5 minutes duration were taken for consideration.

For the case of normal subject, the 5-minute segment were chosen from relatively artifact free zone. For the case of epileptic patients manifesting seizure activity, this 5-minute segment were chosen from such a position where there is a notable amount of seizure activity. The dataset comprises of total 11 subjects, within which 5 are normal subjects and 6 patients were diagnosed with epileptiform activities manifesting focal seizures. The seizure activities lasted on an average of $90 \mathrm{sec}$. Few patients demonstrated multiple seizure epochs in their recordings. However, for the sake of simplicity, we chose to take a segment of 5 minute from pre-ictal period to post-ictal period which contains the most prominent seizure activity. On the other hand, 5 minutes segment from normal subjects were chosen from the effectively clean duration of recording.

Beside the controls and the patients, another subject was chosen, who was clinically diagnosed not to have any sort of neuro abnormalities. The EEG segments acquired from this subject is termed as template. Clinical experts verified the data segment and reported not to have any sort of abnormalities in this template data. This template will be used as a standard of comparison in our study.

\section{Methods}

The processing of EEG data is very complex and cumbersome as these signals are non-stationary in nature and have very low signal to noise ratio. Furthermore, these EEG signals, tend to get corrupt with different type of artifacts evolving from eye blinks, eye movement, muscle movement and cardiac activities. The steps involved in processing of the EEG signal are described further. 


\subsection{Filtering and Denoising}

Our preliminary approach starts with designing a digital bandpass Butterworth filter of order 6 which is a cascaded low pass and high pass filter with high cut off $40 \mathrm{~Hz}$ and low cut off $0.05 \mathrm{~Hz}$. The filter ranges are so chosen to get all the sub band frequency components of the EEG signal. All 21 channels of the resultant EEG signal are subjected to the denoising process. However, the sampling rate was kept constant at $250 \mathrm{~Hz}$ to maintain the integrity of the signal.

\subsection{Discrete Wavelet Transform}

Wavelet Transform (WT) is a powerful mathematical tool which can express the EEG signal in multiscale time-frequency domain and able to capture subtle changes in the signal [13]. In this work, we Daubechies $4(\mathrm{db} 4)$ wavelet is selected because several studies have suggested the use of this wavelet in terms of its smoothing feature and sensitive to detect the random changes in EEG signal [14]. Daubechies wavelet family are the most eloquent wavelet which finds uses in numerous applications [15]. Basically, in Discrete Wavelet Analysis, the decomposition of a given signal $x(t)$ into its consequently increasing finer detail is given by two basic functions, the wavelet can be represented as in equation-

$$
x(t)=\sum_{k} 2^{\frac{j 0}{2}} a_{j 0}(k) \varphi\left(2^{j 0} t-k\right)+\sum_{j=j 0}^{\infty} \sum_{k} 2^{\frac{j}{2}} d_{j}(k) \psi\left(2^{j} t-k\right)
$$

where function $\varphi(t)$ and $\psi(t)$ are the scale factors and mother wavelet respectively. In the above equation first summation represents an approximate of $x(t)$ with scale index $j 0$, while the second summation adds more detail using fine scales of $j$. Thus computed $x(t)$ is the coefficient of discrete wavelet transform. The energy spectrum of the wavelet is given by:

$$
|\psi(f)|^{2}=2 \pi^{\frac{1}{2}} e^{-\left(2 \pi f-2 \pi f_{0}\right)^{2}}
$$

Sampling rate of the signal directly relates to the frequency band of each detail level, which is given by $f_{m}=f_{s} / 2^{l+1}$ where $f_{s}$ is the sampling frequency and $l$ is the required level of decomposition. In this work the sampling frequency is $250 \mathrm{~Hz}$ and hence according to Nyquist theorem, highest frequency contained in the signal would be therefore $f_{s}=2$. The signal is decomposed into 6 level in our case i.e., from D1-D6 representing the detail coefficients and A6 representing the approximate coefficient. Among these D4 represents Beta band $(15-30 \mathrm{~Hz})$ of the EEG signal.

\subsection{Wavelet Coherence and Continuous Wavelet Transform}

The continuous wavelet transform (CWT), denoted by $W(s, \tau)$ is computed as convolution of EEG signal $x(t)$ with a wavelet function $\phi_{S, \tau}$ as denotes the cwt function of a signal.

$$
W(s, \tau)=\int_{-\infty}^{+\infty} x(t) \psi_{s, \tau}^{*}(t) d t
$$


where $\mathrm{s}$ is the time scale and $\tau$ is the time shifted version of the wavelet transformation.

In CWT, the signal being analyzed is matched and convolved with the chosen wavelet basis function. The transform is done in continuous time and frequency increments. The wavelet basis function, $\psi_{s, \tau}$ in equation 1.3 represents a member of the mother wavelet $\phi_{0}$. The mother wavelet may real or can be complex conjugate.

$|\psi(f)|^{2}=2 \pi^{\frac{1}{2}} e^{-\left(2 \pi f-2 \pi f_{0}\right)^{2}}$

In CWT, time-frequency resolution can be adjusted by changing the inner parameters of the scalable wavelet function. For this work, we chose scale as 19 which gives better frequency resolution response to the beta band. Further, choice of wavelet basis function, as in case of DWT, also forms an important point in degree of correlation of the wavelet with the signal. We choose morlet wavelet as our preferred wavelet for the resultant CWT analysis and wavelet coherence feature.

Morlet wavelet is a complex function represented by real and imaginary part. Morlet have advantages that it gives both amplitude and phase information of the signal. The wavelet is represented as:

$\psi(t)=\pi^{-\frac{1}{4}} e^{i 2 \pi f_{0} t} e^{-\frac{t^{2}}{2}}$

The wavelet can be thought of a complex wave with a Gaussian envelope $\left(e^{-\frac{t^{2}}{2}}\right)$.The normalization factor of the wavelet entitles the wavelet $\left(\pi^{-\frac{1}{4}}\right)$ function for unit energy.

The resultant wavelet coefficient calculated from the continuous wavelet transform is then computed with the template signal as Wavelet Coherence. The CWT of the EEG signals from the normal subject and patient forms the basis of wavelet coherence with that of the CWT coefficient thus calculated from the template subject. Since we intent to calculate the phase and modulus of the degree of coherence between the beta band during evolution of seizure, the onset and the seizure free zone, we try to obtain the spatial resolution by using the wavelet coherence (wcoh) between the template and the above three classes. Coherence is used to find the relationship between two input signals by dividing the squared magnitude of the cross-spectral density of two signals by the product of their power spectral densities:

$$
C_{x y}(f)=\frac{\left|P_{x y}^{2}(f)\right|}{P_{x x}(f) P_{y y}(f)}
$$

where $f$ is frequency, $P_{x x}$ and $P_{y y}$ represent the power spectral densities of the two signal $x$ and $y$ respectively [16]. $P_{x y}$ is computed as cross spectral density of the two-input signal $x$ and $y$ is obtained by product of the Fourier transform of signal ${ }^{x}$ and the complex conjugate of the Fourier transform of the other signal ${ }^{y}$. The values of coherence ranges from 0 to 1 . Higher coherence value depicts the two signals are close match to each other and have similar spectral densities. For values pertaining to one, the signals are an exact match. As EEG signals are non-stationary signals the coherence is calculated as therefore time-varying spectral analysis, such as CWT is used in accordance to wavelet coherence.

\subsection{Wavelet Cross Spectrum}


The wavelet cross spectrum of two input signals $\mathrm{x}$ and $\mathrm{y}$ are computed as:

$C_{x y}(a, b)=S\left(C_{x}(a, b) C_{y}^{*}(a, b)\right)$

where $C_{x}(a, b)$ and $C_{y}(a, b)$ represents the wavelet transforms of the two signal $x$ and $y$ at scales $a$ and position $b$. The function is used to generate the cross spectrum of the two-input signal after their wavelet coherence analysis. the mechanism of wavelet cross spectrum analysis supports the fact that the frequency spectrum of the wavelet closely matches with that of the spectrum of the two signals. The identity is computed using wcoher function in MATLAB.

\subsection{Three-way PARAFAC Analysis}

In standard multivariate data, like that of EEG signal features, datas are arranged in two-way structure- a table or a matrix. Independent Component Analysis (ICA) and Principal Component Analysis (PCA) are used to decompose these data into feature matrices. However, for large dataset like that of EEG, it is cumbersome and hence it is appropriate to use three-way array or table to map the feature components. Here the matrices are considered of size $I \times J \times K$. This forms the basis of a multiway array. There are several models which fits these multiway array structure to decompose into components. Multiway models are popular for decomposing EEG data into space-time-frequency components [17]. Here we use parallel factorization (PARAFAC) model to decompose the wavelet coherence coefficients into 4 components. We use the PARAC model as was described by Harshman et al. in their work on handling multiway data [18]. The basic structural model for decomposition of the data matrix $S_{\left(N_{d} \times N_{f} \times N_{t}\right)}$ (where $N_{d}, N_{f}$ and $N_{t}$ are the number of channels, scales of frequency and time points respectively obtained from the wavelet transformation) for the elements $S_{d f t}$ can be defined by: $\bar{S}_{d f t}=\sum_{k=1}^{N_{k}} a_{d k} b_{j k} c_{t k}$

The idea behind this equation is to find loading matrices $\mathrm{A}, \mathrm{B}, \mathrm{C}$ where the constituent elements are $a_{d k}$, $b_{j k}$ and $c_{t k}$ respectively. In our work, we use $k$ components which is also termed as an atom, to map the corresponding elements and thus the atoms will be $a_{k}, b_{k}$ and $c_{k}$ respectively. These are again represented as spatial, spectral and temporal signatures for the atoms. The prime advantage of this method is that it decomposes the time varying, non-stationary EEG spectrum to atoms for easy analysis of the signatures. The model has also advantage of providing high signal-to noise ratio if the number of components chosen are appropriate. A powerful approach in selecting the number of components in parafac is the Core Consistency Diagnostic (Corcondia) that can select appropriate number of components. We have used this method of for selection of the number of components. However, one drawback of the PARAFAC model is that it requires careful preprocessing of the data. We have utilized this algorithm to first compute the atoms A, B, C for the normal subject with seizure free EEG data and then to the epileptic subjects with 3 classes of EEG data (seizure -free, onset and seizure). The output atom $\mathrm{A}$ is the spatial component which is classified to precisely localize the three classes (seizure free, onset and seizure).

\subsection{Classification of Feature set}


The purpose of the use of classification in this work is to identify the 3 classes i.e., the seizure-free, onset and seizure class. The extracted features are marked with true labels which indicate their true class. The seizure, onset and seizure free data are marked 0,1,2 respectively. The extracted features are given as input for the classifiers: Fine Gaussian SVM, Weighted KNN, Ensemble Bagged Trees and Ensemble Subspace KNN. For the sake of simplicity, the classifiers are marked as:

Classifier 1: Fine Gaussian SVM

Classifier 2: Weighted KNN

Classifier 3: Ensemble Bagged Trees

Classifier 4: Ensemble Subspace KNN

All the classifiers were used with 7-fold cross validation.

\section{Proposed Framework}

In this study, we acquire EEG signals from normal subjects and epileptic patients with marked duration of seizure episodes. We also take another normal subject which was labeled as "template" in our case. We use the template data as a standard reference in building up the feature matrix. As EEG signals have high potentiality for getting corrupt with artifacts, we perform denoising of these signals using digital filters

designed to make the signal noise free. Next, we perform decomposition of the EEG segment into its constituting sub bands by Discrete Wavelet Transform (DWT). Further we perform Continuous Wavelet Transform (CWT), Wavelet Coherence and Cross Spectrum analysis to extract 2-dimensional feature set. This forms the basis of time frequency analysis in our case. This 2-dimensional feature set is transformed to a 3-dimensional matrix with channels $\mathrm{x}$ frequency $\mathrm{x}$ time as its output parameter with the help of a unique tensor decomposition model approach known as Parallel Factorization (PARAFAC). The output of this parafac model forms the input to 3 different classifiers and performance are judged based on the accuracy and Receiver Operating Characteristic curve. Our study tries to focus on normal as well as patients with focal epileptic seizures for classification of 3 classes, i.e., the seizure free, onset and seizure classes. The approach of our work can be represented in blocks as in Figure 1.

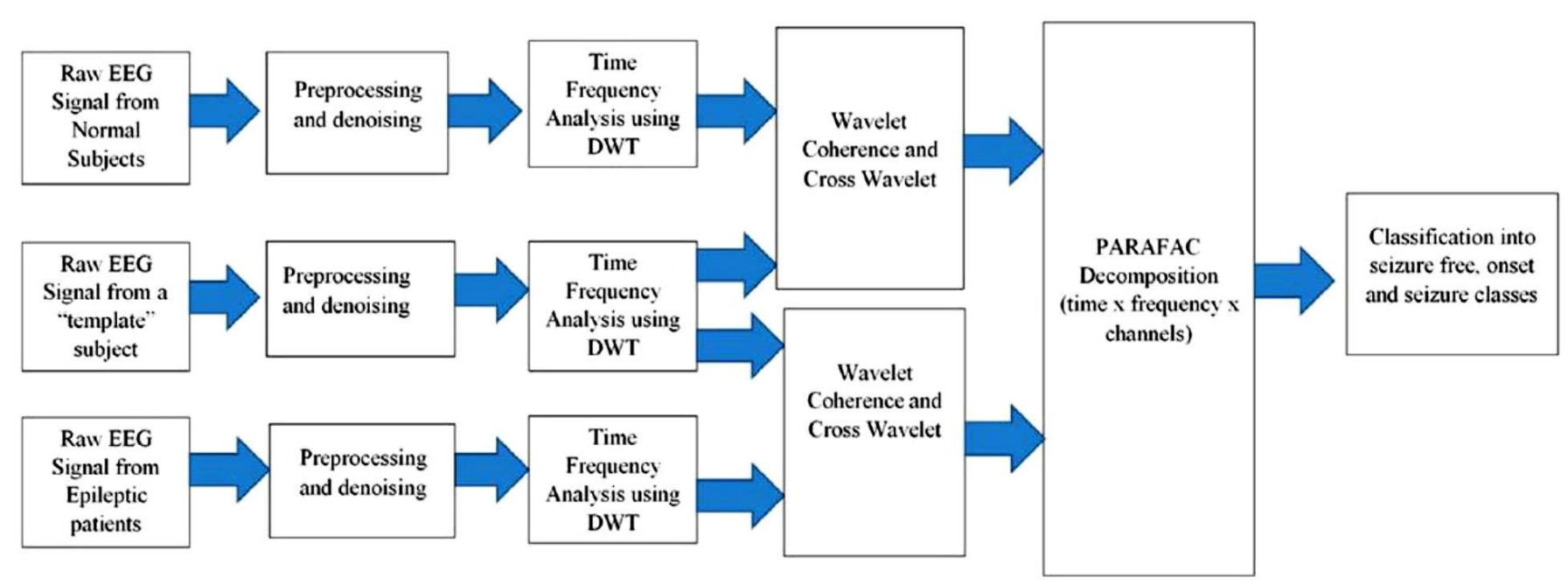

Figure 1: Block diagram of our proposed approach 


\section{Results and Discussions}

In the present approach, 5 min samples EEG dataset containing 12 subjects with 5 normal subject, 6 epileptic subject and 1 normal subject marked as template subject are subjected to preprocessing to remove artifacts from the raw EEG signal. Butterworth Bandpass filtering has been used to de-noise and filter the raw EEG signals. This has been done on the 5 min EEG signals from the patients, subject and the template. Therefore, the filtering influences the individual classes (seizure- free, onset and seizure). A sample $5.35 \mathrm{sec}$. data (corresponding to one window) of the acquired raw EEG signal during seizure period from one of the channels (F7) of one patient and its corresponding filtered signal is shown in Figure $2 a$ and Figure $2 b$ respectively.

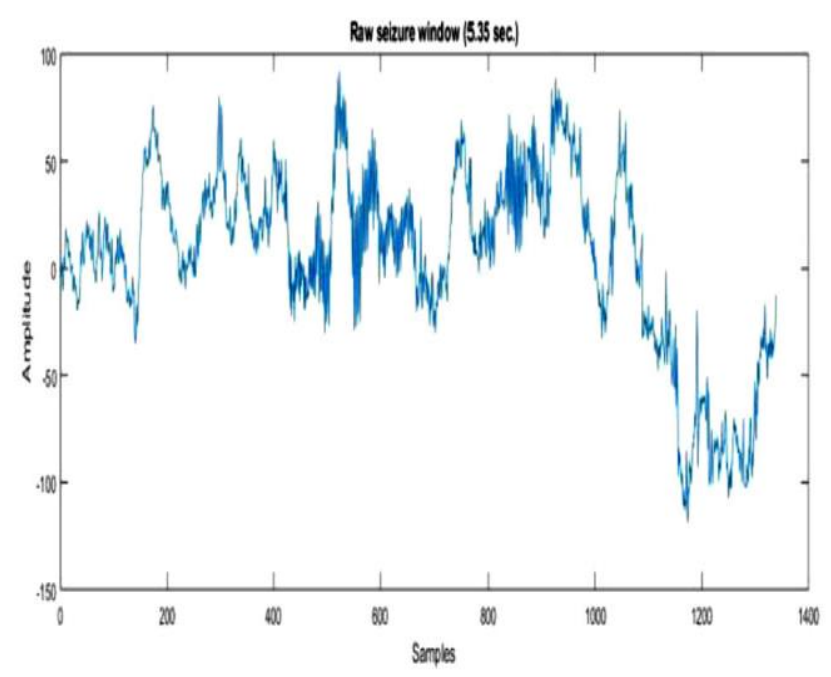

(a)

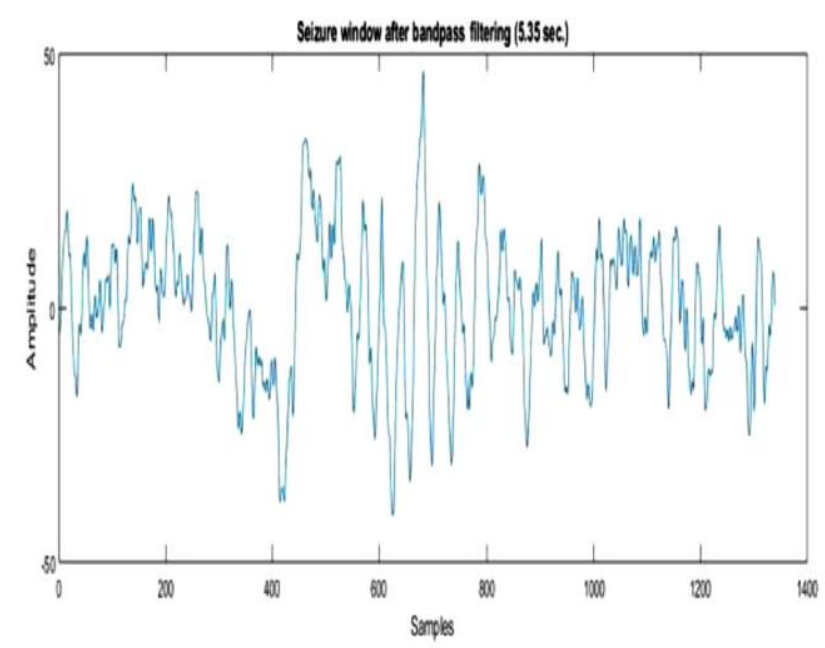

(b)

Figure 2: Pre-processing of seizure window (a) Raw seizure window (b) A seizure window after Bandpass filtering.

The same raw and filtered data of $5 \mathrm{sec}$. epoch at the time of onset of seizure from the same channel (F7) is depicted in Figure $3 a$ and Figure $3 b$ respectively. Clear decrease in amplitude can be visualized from the seizure window.

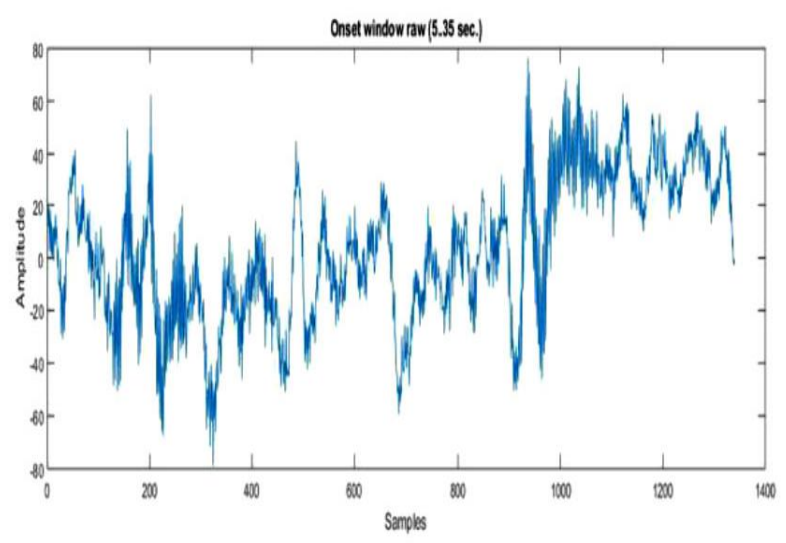

(a)

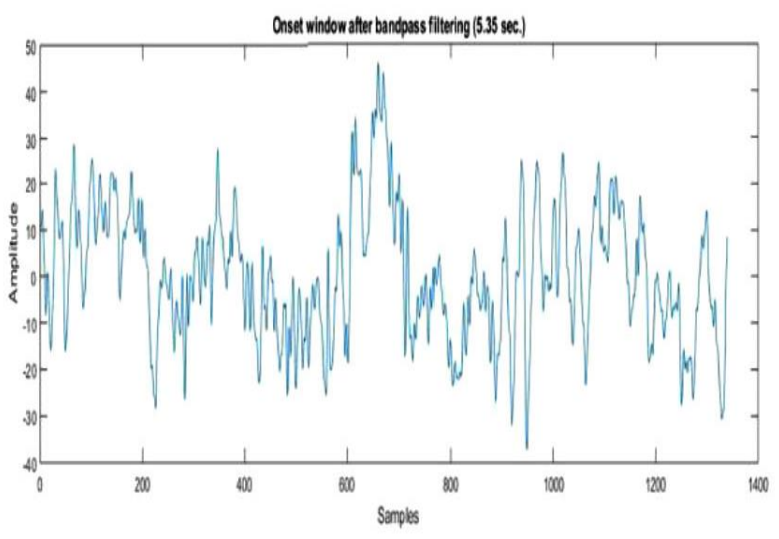

(b)

Figure 3: Pre-processing of onset window (a) Raw onset window (b) An onset window after Bandpass filtering. 
Use of Butterworth bandpass filter removes the high frequency noise which gets corrupted with the EEG signal. Hence the frequency components of the signal remain preserved. As in this case, the high frequency noise is removed preserving the actual frequency component of the signal. A sample window of the seizure free labeled window for both the preprocessing and postprocessing stages shown in Figure $4 \mathrm{a}$ and Figure $4 \mathrm{~b}$ respectively. Further decrease in amplitude can be from the onset samples.

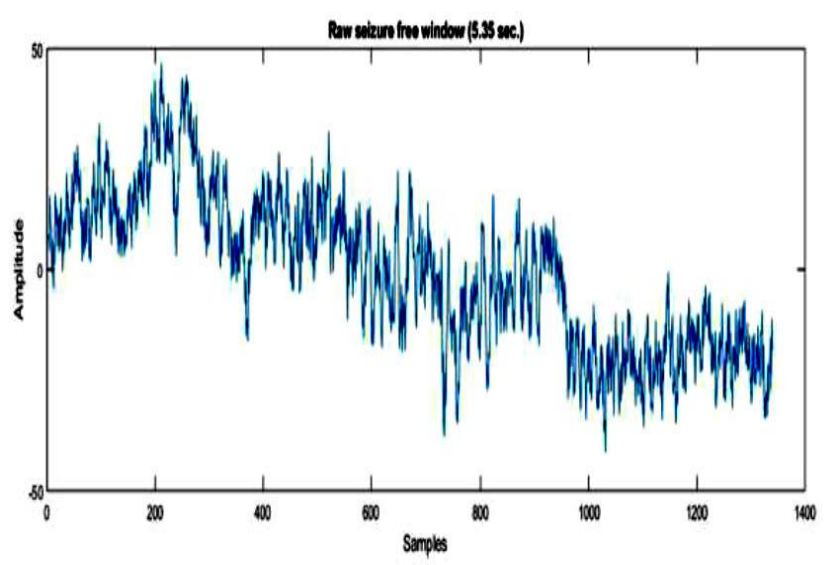

(a)

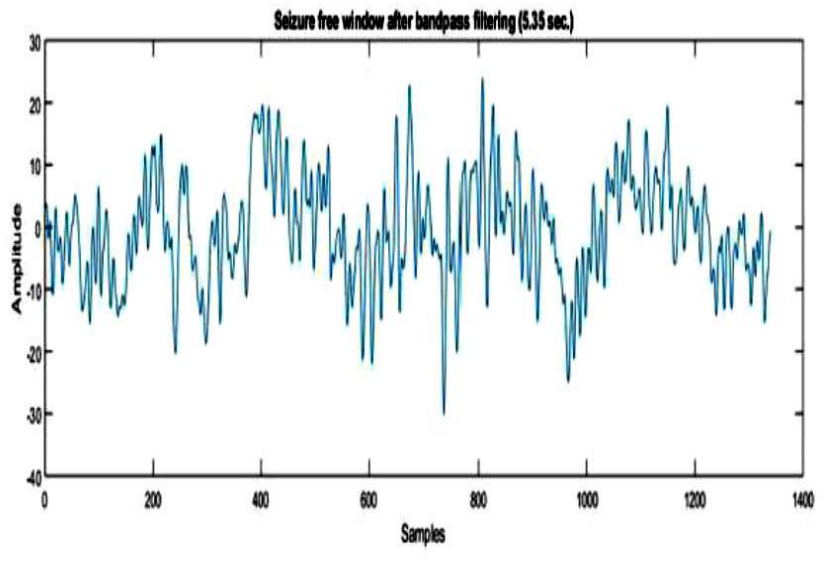

(b)

Figure 4: Pre-processing of seizure-free window (a) Raw seizure-free window (b) A seizure-free window after Bandpass filtering.

In the next step, we perform discrete wavelet transform with Daubechies4 wavelet and decompose till level 6. We take the 4th level detailed sub-band as it corresponds frequency of the beta band of EEG signal. Figure 5 shows a sample window of the beta sub-band acquired from the seizure window. The beta band constitutes the frequency band from $15-30 \mathrm{~Hz}$. DWT uses db4 wavelet to match the wavelet and thus decompose into detail and approximate levels.

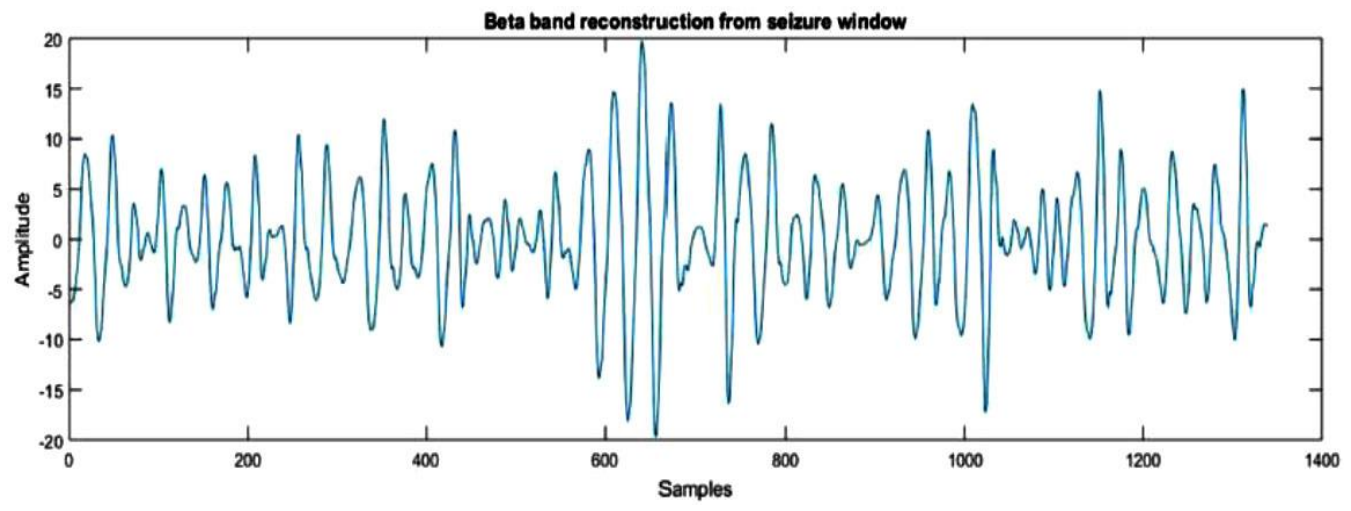

Figure 5: Beta band reconstruction from seizure window

The beta band as reconstructed by DWT during onset period is represented in Figure6. Morphologically the beta band of the onset period differs from the seizure period which forms the key aspect of extraction of feature in the preceding steps. 


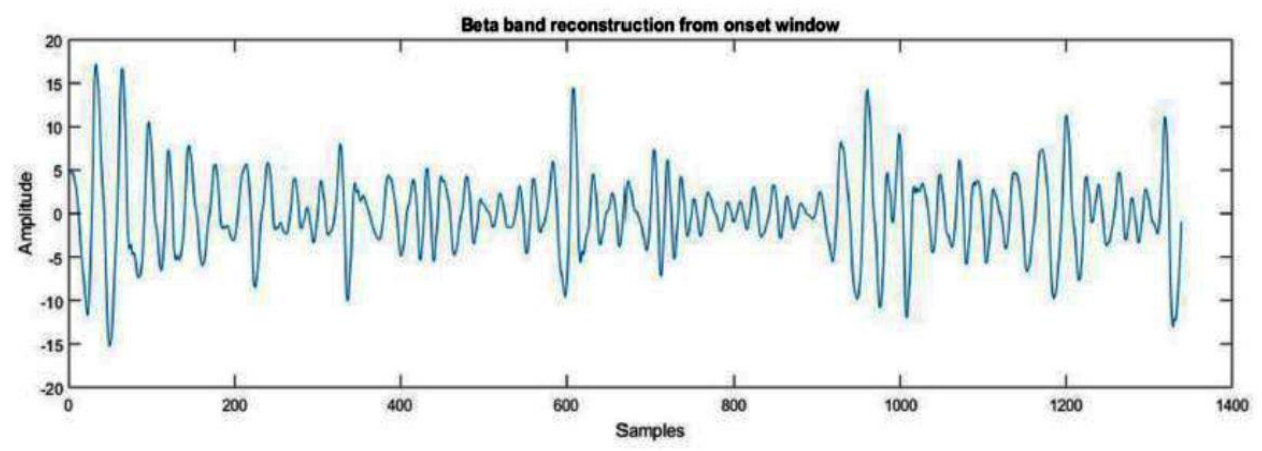

Figure 6: Beta band reconstruction from onset window

The reconstructed beta band for a seizure-free interval can be seen in Figure7. The morphological differences from the seizure interval and the normal interval can be well observed.

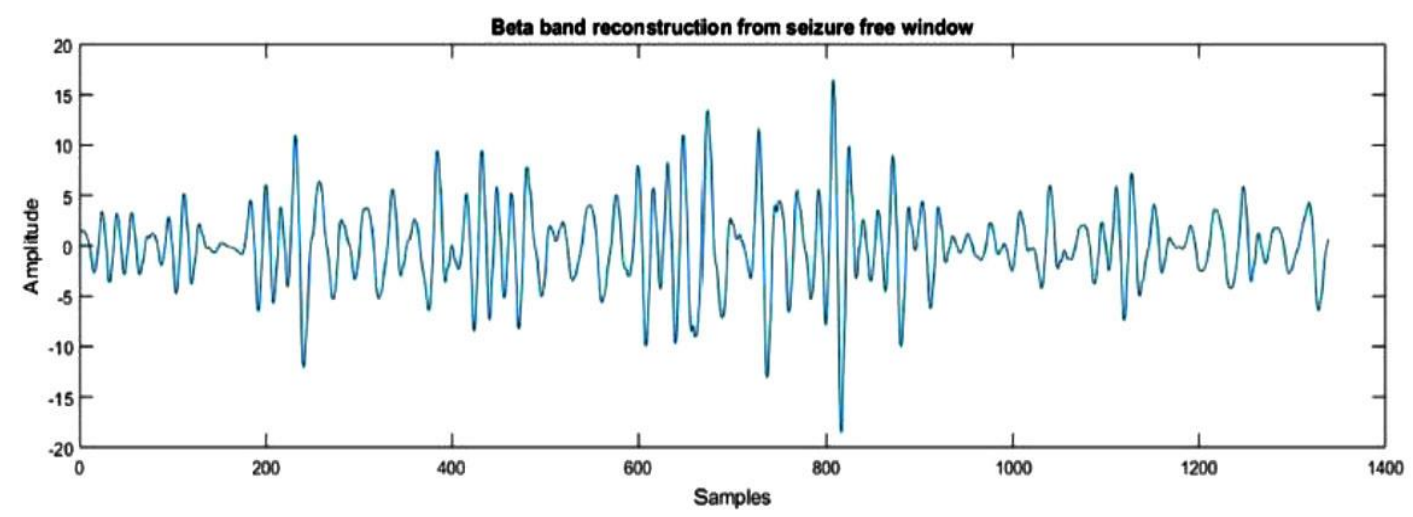

Figure 7: Beta band reconstruction from seizure-free window

We perform wavelet decomposition on the filtered template signal as well. Figure8 shows the reconstructed beta band from one window of the template signal.

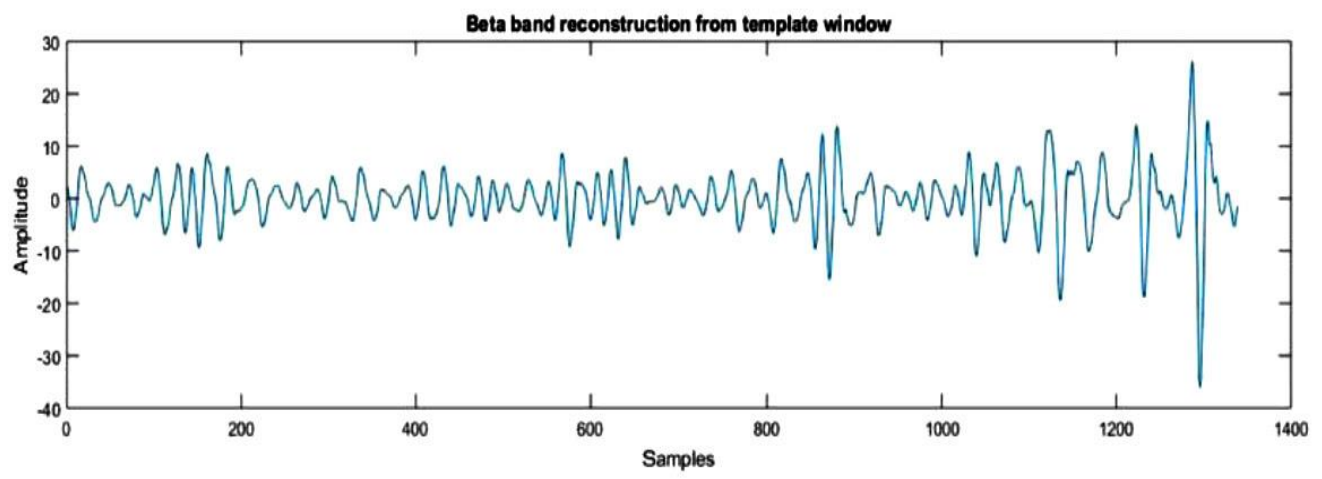

Figure 8: Beta band reconstruction from template window

Further after getting the beta band from the filtered EEG epochs from each channel and each window, our algorithm performs the continuous wavelet transform of the beta band of the seizure-free, onset and seizure interval and the beta band of the template signal. Each of these figures are illustrated in below. Figure9 represents CWT of the beta band of template and seizure free window. 

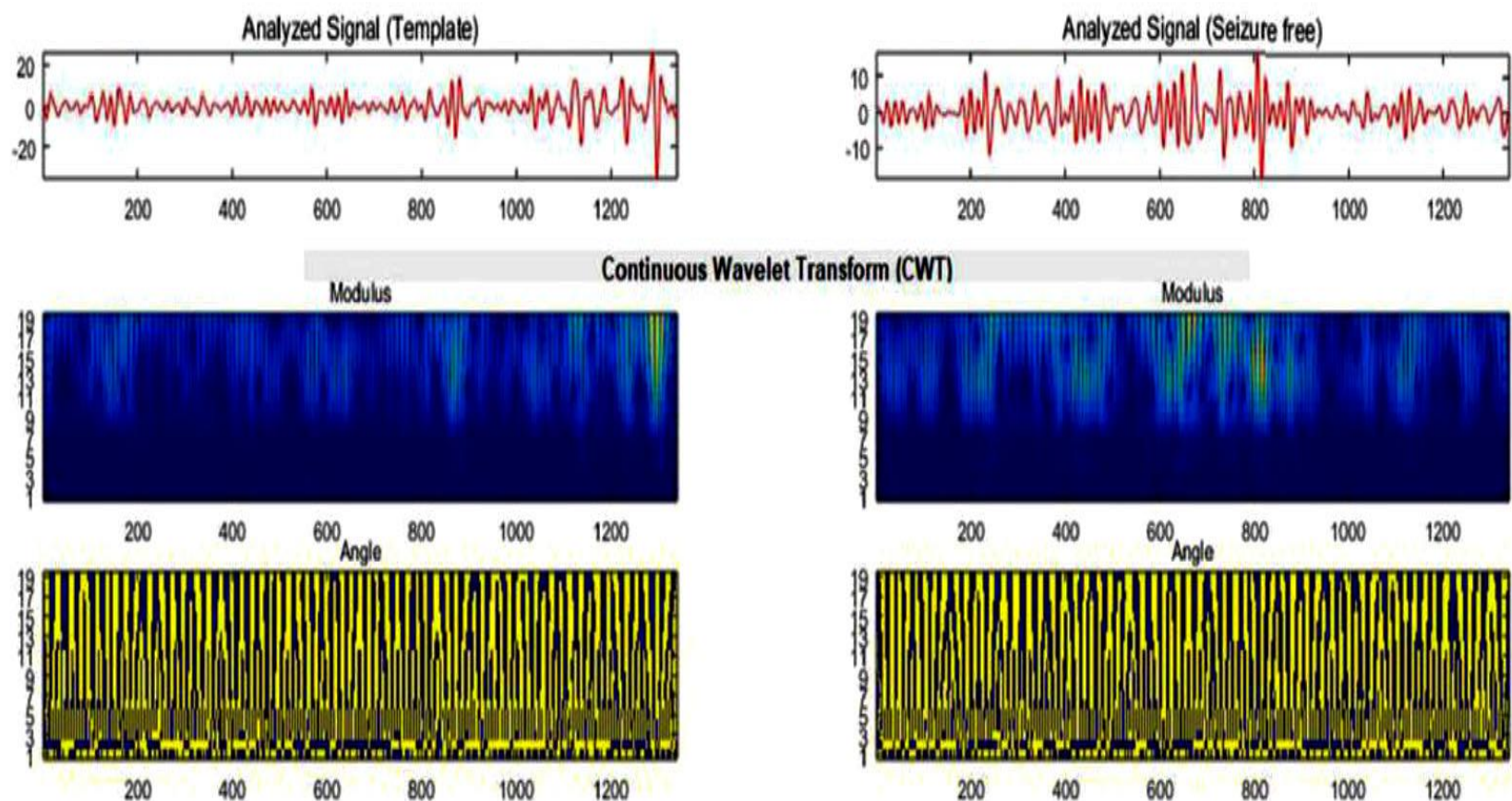

Figure 9: CWT for template and seizure-free

Figure10 represents the CWT for template and seizure window. The differences in modulus for both analyzed signal can be seen from the figures 9 and 10 .
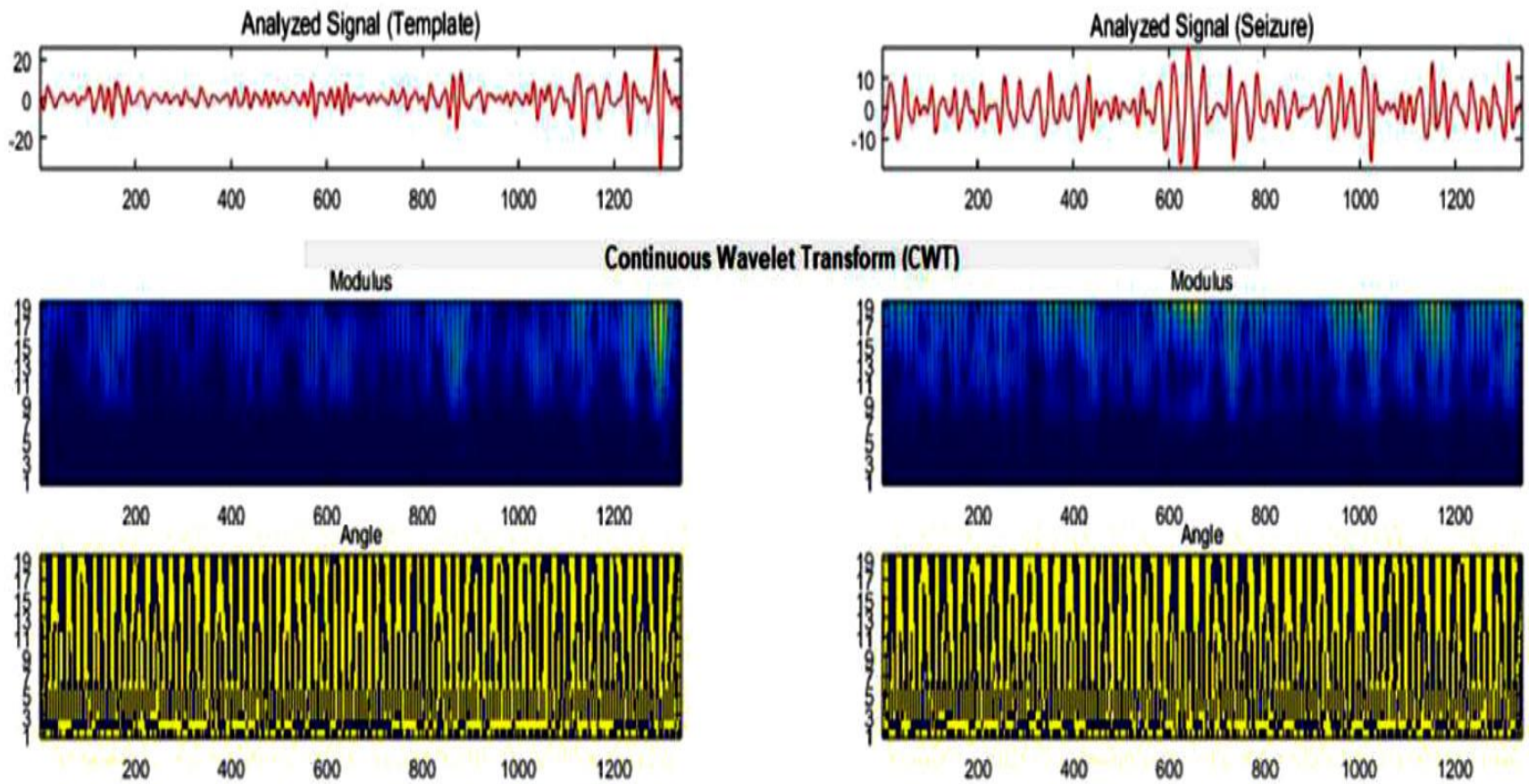

Figure 10: CWT for template and seizure 
The CWT matches and convolves the chosen wavelet basis function with that of the analyzed signal. Here as morlet is selected as wavelet basis function, it performs the wavelet transforms accordingly. Next the wavelet coherence features are computed for each of the three intervals with respect to the template class. Morlet wavelet is used to compute the coherence between each of these. In Figure 11 the wavelet coherence between a seizure-free and template window can be seen. The wavelet coherence measures the coherence on a scale ranging from 0 to 1 . The more the coherence, we get more of the coherence coefficients. This is done for all the frequency scales. The number of scales here is 19 and hence it is seen that at the maximum zone of coherence all the 19 scales have same coefficient values.

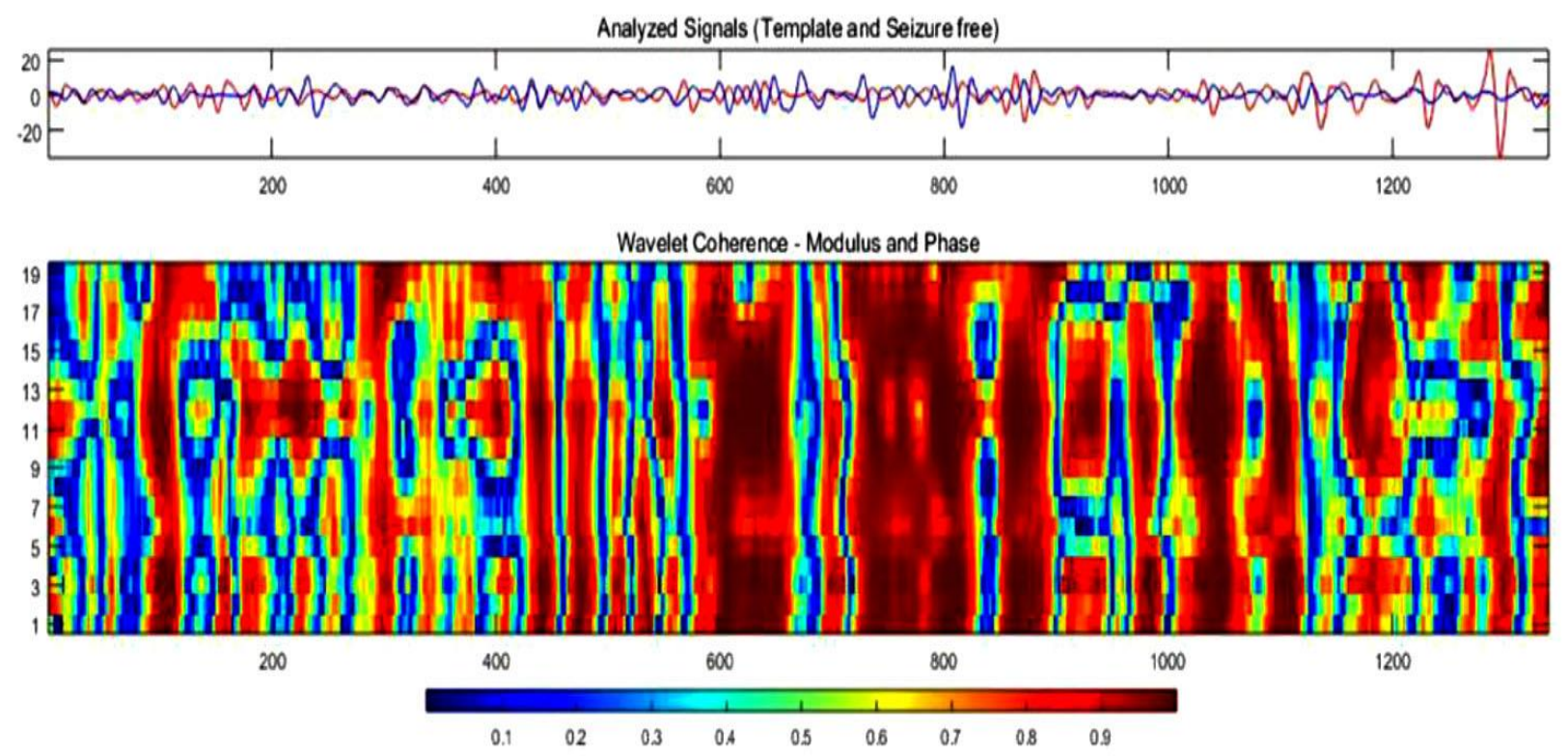

Figure 11: Wavelet Coherence for a template and a seizure-free window

Further we compute the wavelet coherence for the template window and the seizure window. The difference in wavelet coherence values can be well observed from that of the template and the seizurefree interval. Figure12 shows the coherence for template and seizure window.
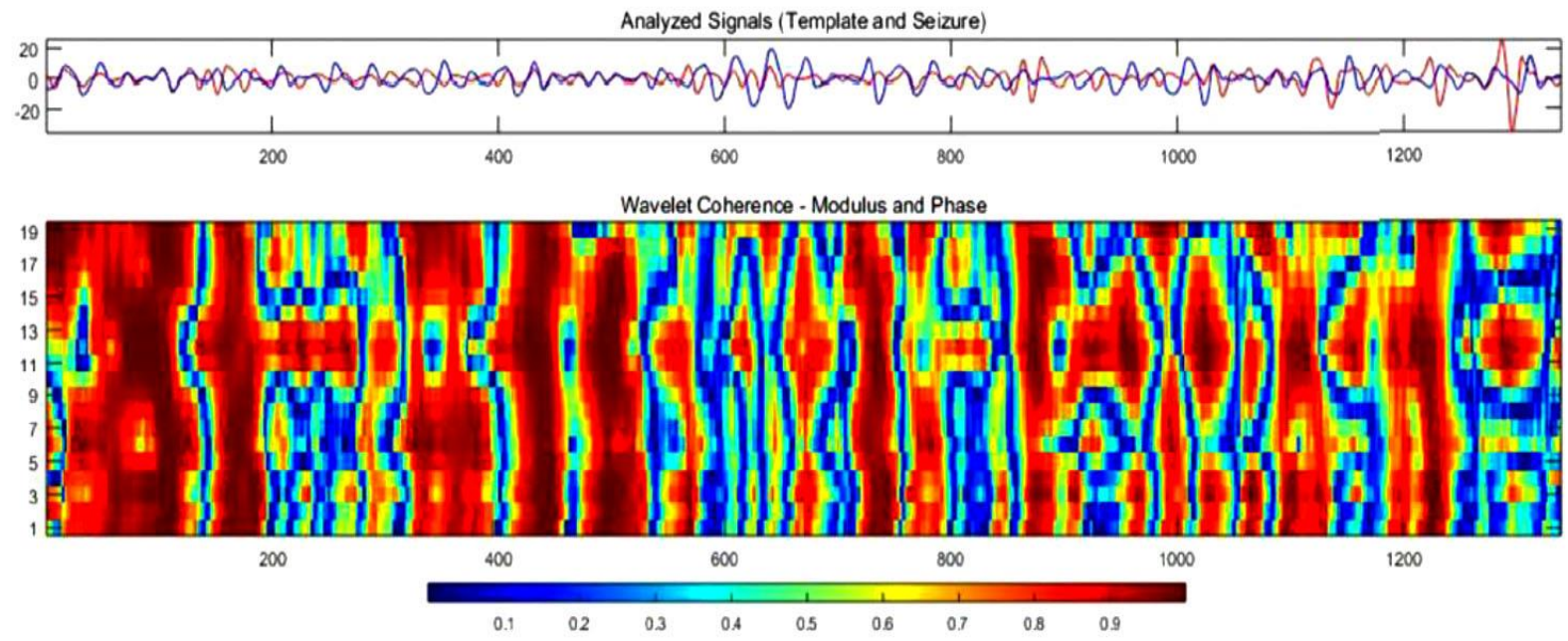

Figure 12: Wavelet Coherence for a template and a seizure window 
Wavelet coherence features forms the basis of differentiation between the seizure free and seizure class. The modulus and phase of wavelet coherence are the deterministic characteristics for evaluation of these signals. Wavelet Cross Spectrum (WCS) features are computed along with the wavelet coherence features. The Cross-Spectrum features for template and seizure free are illustrated in Figure13.
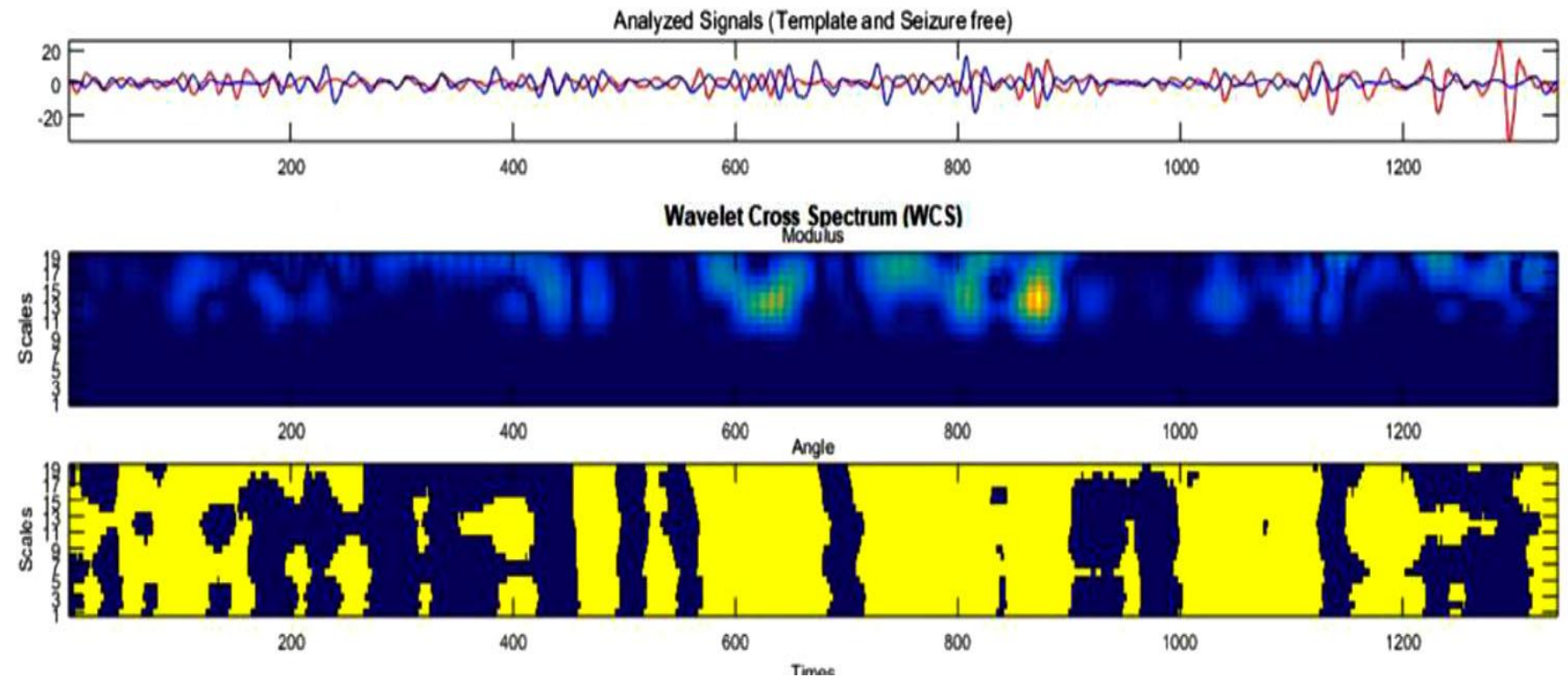

Figure 13: Wavelet Cross Spectrum for a template and a seizure-free window

The wavelet cross spectrum for template and seizure window are also computed. Figure14 shows the WCS for these two windows. The modulus and angles are plotted as functions of scale values which again represents the frequency.
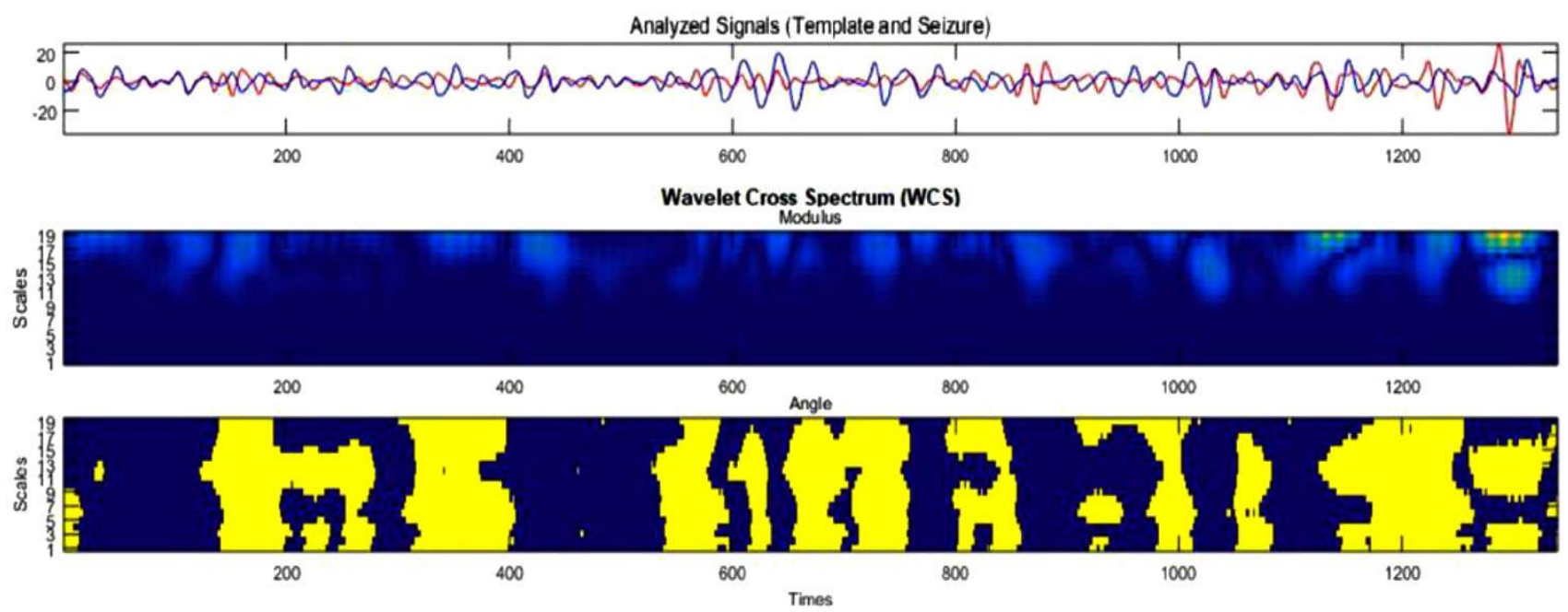

Figure 14: Wavelet Cross Spectrum for a template and a seizure window

Finally, the evaluated features (WCOH and WCS) are taken into consideration for three-way array input as a function of time $\times$ frequency $\times$ channels and PARAFAC analysis has been performed. The output matrices are $\mathrm{A}, \mathrm{B}$ and $\mathrm{C}$ denoting 3 different loadings. As we have performed PARAFAC analysis for 4 components we got 4 different predictors for classification according to the true levels of the classes. The true classes were marked as $0=$ Seizure, $1=$ Onset, $2=$ Seizure-free intervals. Accordingly, we the 
machine learning approach used for 4 different classifiers compute individual accuracy, specificity, sensitivity, F score and Area Under the Curve (AUC) taking seizure class as the true class. The computed parameters for the classification of the feature set $\mathrm{WCOH}$ are shown in Table1. The classification performances show Classifier3 (Ensemble Bagged Trees) has highest accuracy in predicting the seizure class from the $\mathrm{WCOH}$ feature.

Table 4.1: Classification performance of Wavelet Coherence features for Seizure as true class

\begin{tabular}{|l|l|l|l|l|l|}
\hline \multirow{2}{*}{ Classifiers } & \multicolumn{4}{l}{ Classification Performance } \\
\cline { 2 - 6 } & $\begin{array}{l}\text { AUC } \\
(\%)\end{array}$ & Accuracy $(\%)$ & $\begin{array}{l}\text { Specificity } \\
(\%)\end{array}$ & $\begin{array}{l}\text { Sensitivity } \\
(\%)\end{array}$ & F-score \\
\hline Classifier1 & 71.56 & 81.70 & 97.93 & 14.47 & 0.2316 \\
\hline Classifier2 & 78.66 & 81.10 & 94.42 & 29.42 & 0.3726 \\
\hline Classifier3 & 81.20 & 82.21 & 95.70 & 28.99 & 0.3627 \\
\hline Classifier4 & 69.69 & 79.42 & 94.47 & 19.66 & 0.2660 \\
\hline
\end{tabular}

The classification performance output of wavelet cross spectrum feature is evaluated and shown in Table 4.2. Classifier 3(Ensemble Bagged Trees) has highest accuracy in predicting the seizure class from the WCS feature.

Table 4.2: Classification performance of Wavelet cross spectrum features for Seizure as true class

\begin{tabular}{|l|l|l|l|l|l|}
\hline \multirow{2}{*}{ Classifiers } & \multicolumn{4}{l}{ Classification Performance } \\
\cline { 2 - 6 } & $\begin{array}{l}\text { AUC } \\
(\%)\end{array}$ & Accuracy $(\%)$ & $\begin{array}{l}\text { Specificity } \\
(\%)\end{array}$ & $\begin{array}{l}\text { Sensitivity } \\
(\%)\end{array}$ & F-score \\
\hline Classifier1 & 71.58 & 84.29 & 97.97 & 14.57 & 0.2332 \\
\hline Classifier2 & 78.79 & 81.31 & 94.70 & 29.14 & 0.3732 \\
\hline Classifier3 & 81.10 & 84.76 & 95.78 & 28.52 & 0.3803 \\
\hline Classifier4 & 69.73 & 82.07 & 94.28 & 19.80 & 0.2658 \\
\hline
\end{tabular}

Receiver Operating Characteristic curve (ROC) curves are also evaluated for these 3 classes. ROC illustrates the diagnostic ability of a binary classifier system by varying its discrimination threshold. It is as the true positive rate (TPR) against the false positive rate (FPR) at various threshold settings. The 
ROC curve for Classifier 3 (Ensemble Bagged Trees) for classification of the wavelet coherence feature has been shown in Figure15 for all the 3 classes.

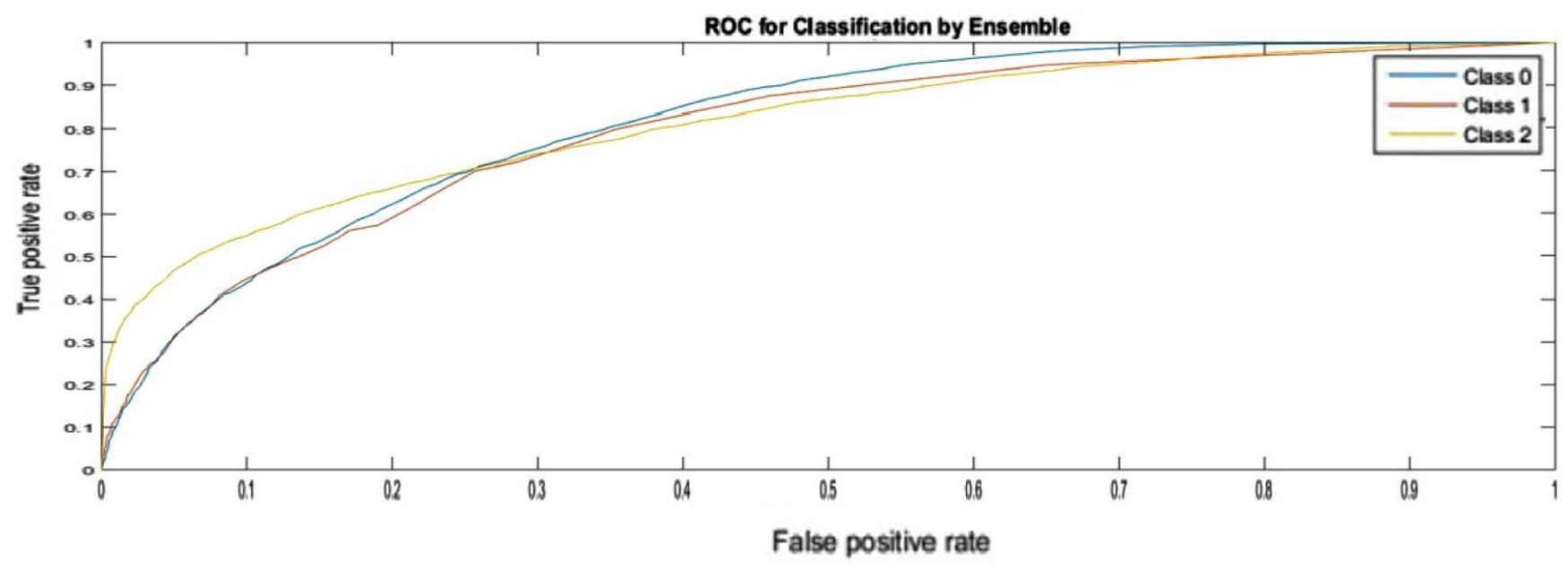

Figure 15: ROC curve for Classifier 3 (Ensemble Bagged Trees) for classification of the wavelet coherence feature

Again, the ROC curve for Classifier 3(Ensemble Bagged Trees) for classification of the wavelet cross spectrum feature has been shown in Figure16 for all the 3 classes.

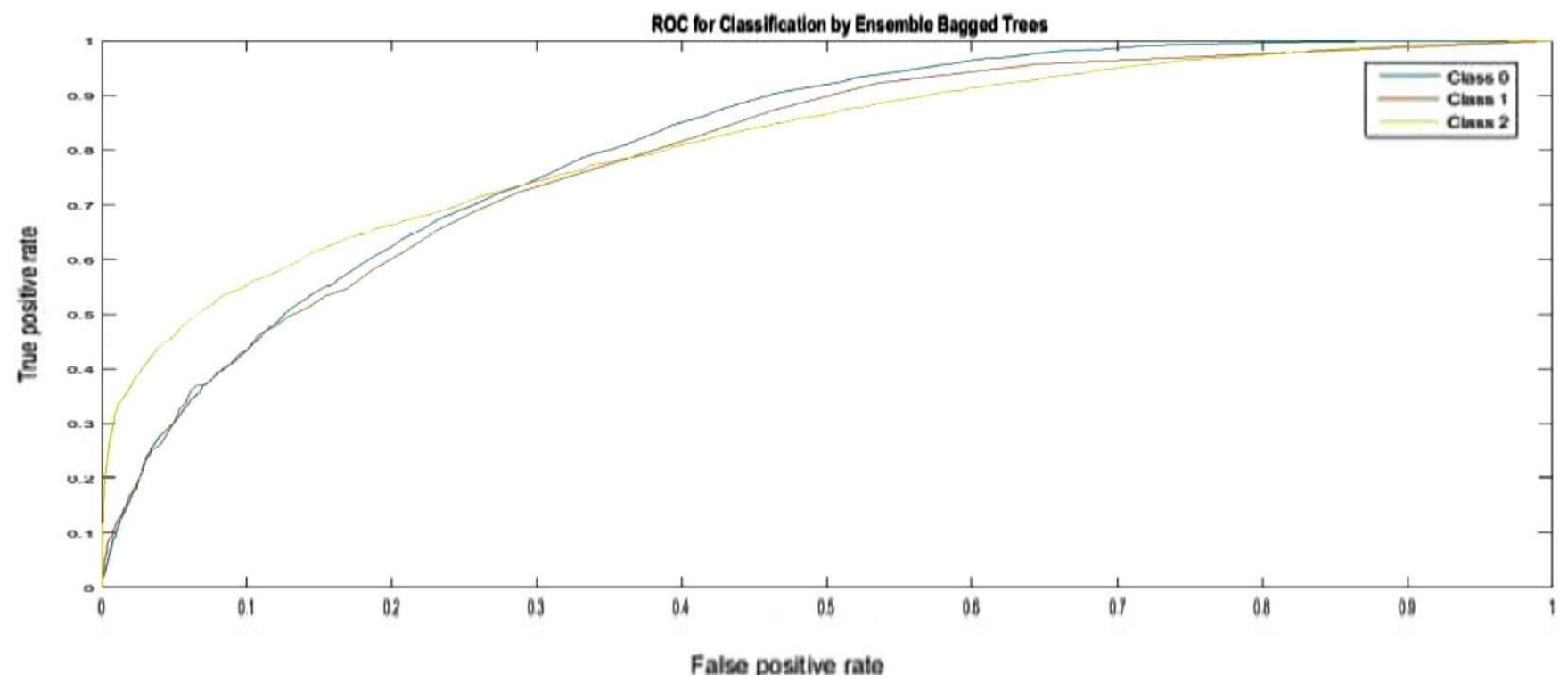

Figure 16: ROC curve for Classifier 3 (Ensemble Bagged Trees) for classification of the wavelet cross spectrum feature

Thus, Ensemble Bagged Trees classifiers are best suited to classify the WCOH and WCS feature for the proposed method.

\section{Conclusion}

Detection of epileptic seizure prior to their occurrence is a matter of great challenge. Electroencephalogram based study also proved its potential efficiency to detect seizure using wavelet 
analysis and decomposition of features into multi-way space-time-frequency localized data using a unique canonical decomposition approach (PARAFAC). Wavelet Transforms and analysis proves its superiority over other methods of feature generation. PARAFAC model approach depicts the suitability of using it as a prospective tool in handling huge multi-dimensional data sets. The multi-way canonical approach used in this work effectively concentrates the features into multi-components thereby reducing the vast data points to many folds. These data points are even easy to classify using several classifiers with good accuracy. Out of these, when utilized the Ensemble Bagged Trees algorithm, we achieved classification accuracy of $84.32 \%$, to classify the features as seizure classes. We may draw conclusion, that our approach proposes to create sharp distinctions between the above three classes to effectively ease the process of detecting an epileptic seizure.

\section{Declarations}

- Availability of data and materials

The clinical dataset for the present work was acquired form Long term EEG monitoring Lab at AMRI Hospital, Kolkata, India.

- Competing interests

The authors declare that they have no competing interests.

- Funding

No funding is available for this research work.

- Authors' contributions

AG analyzed and interpreted the patient data regarding the EEG data and was one major contributor in writing the manuscript. PP was a contributor in writing the manuscript. PB performed the overall supervision for the research work and manuscript. AS performed the planning, supervision and validation of the epileptic seizure detection and was a contributor in writing the manuscript. All authors read and approved the final manuscript

- Acknowledgements

Not applicable

\section{References}

[1] Fisher, R. S., Boas, W. V. E., Blume, W., Elger, C., Genton, P., Lee, P., \& Engel Jr, J. (2005). Epileptic seizures and epilepsy: definitions proposed by the International League Against Epilepsy (ILAE) and the International Bureau for Epilepsy (IBE). Epilepsia, 46(4), 470-472.

[2] Healthline Kimberly Holland. Epilepsy by the numbers: Facts, statistics, and you. http://www.healthline.com/health/epilepsy/ facts-statistics-infographic. Accessed: 2017-05-20.

[3] Kharbouch, A. A. (2012). Automatic detection of epileptic seizure onset and termination using intracranial eeg (Doctoral dissertation, Massachusetts Institute of Technology).

[4] Fisher, R. S., Boas, W. V. E., Blume, W., Elger, C., Genton, P., Lee, P., \& Engel Jr, J. (2005). Epileptic seizures and epilepsy: definitions proposed by the International League Against Epilepsy (ILAE) and the International Bureau for Epilepsy (IBE). Epilepsia, 46(4), 470-472.

[5] Fisch, B. J. (1999). Fisch and Spehlmann's EEG Primer: Basic Principles of Digital and Analog EEG, $3 e$. Amsterdam: Elsevier.

[6] Gotman, J. (1982). Automatic recognition of epileptic seizures in the EEG. Electroencephalography and clinical Neurophysiology, 54(5), 530-540.

[7] Qu, H., \& Gotman, J. (1997). A patient-specific algorithm for the detection of seizure onset in long-term EEG monitoring: possible use as a warning device. IEEE transactions on biomedical engineering, 44(2), 115-122. 
[8] Greene, B. R., Faul, S., Marnane, W. P., Lightbody, G., Korotchikova, I., \& Boylan, G. B. (2008). A comparison of quantitative EEG features for neonatal seizure detection. Clinical Neurophysiology, 119(6), 1248-1261.

[9] UR, A. (2013). Automated diagnosis of epileptic electroencephalogram using independent component analysis and discrete wavelet transform for different electroencephalogram durations. Proceedings of the Institution of Mechanical Engineers, Part H: Journal of Engineering in Medicine, 227(3), 234-244.

[10] Xie, S., \& Krishnan, S. (2013). Wavelet-based sparse functional linear model with applications to EEGs seizure detection and epilepsy diagnosis. Medical \& biological engineering \& computing, 51(1-2), 49-60.

[11] Chen, G. (2014). Automatic EEG seizure detection using dual-tree complex wavelet-Fourier features. Expert Systems with Applications, 41(5), 2391-2394.

[12] Martis, R. J., Acharya, U. R., Tan, J. H., Petznick, A., Yanti, R., Chua, C. K., ... \& Tong, L. (2012). Application of empirical mode decomposition (EMD) for automated detection of epilepsy using EEG signals. International journal of neural systems, 22(06), 1250027.

[13] Adeli, H., Zhou, Z., \& Dadmehr, N. (2003). Analysis of EEG records in an epileptic patient using wavelet transform. Journal of neuroscience methods, 123(1), 69-87.

[14] Subasi, A. (2007). EEG signal classification using wavelet feature extraction and a mixture of expert model. Expert Systems with Applications, 32(4), 1084-1093.

[15] Daubechies, I. (1992). Ten lectures on wavelets (Vol. 61). Siam.

[16] White, L. B., \& Boashash, B. (1990). Cross spectral analysis of nonstationary processes. IEEE Transactions on Information Theory, 36(4), 830-835.

[17] Fumikazu Miwakeichi, Eduardo Martinez-Montes, Pedro A Valdies-Sosa, Nobuaki

Nishiyama, Hiroaki Mizuhara, and Yoko Yamaguchi. Decomposing eeg data into space time frequency components using parallel factor analysis. Neurolmage, 22(3):1035\{1045, 2004.

[18] Harshman, R. A. (1970). Foundations of the PARAFAC procedure: Models and conditions for an" explanatory" multimodal factor analysis. 
Figures

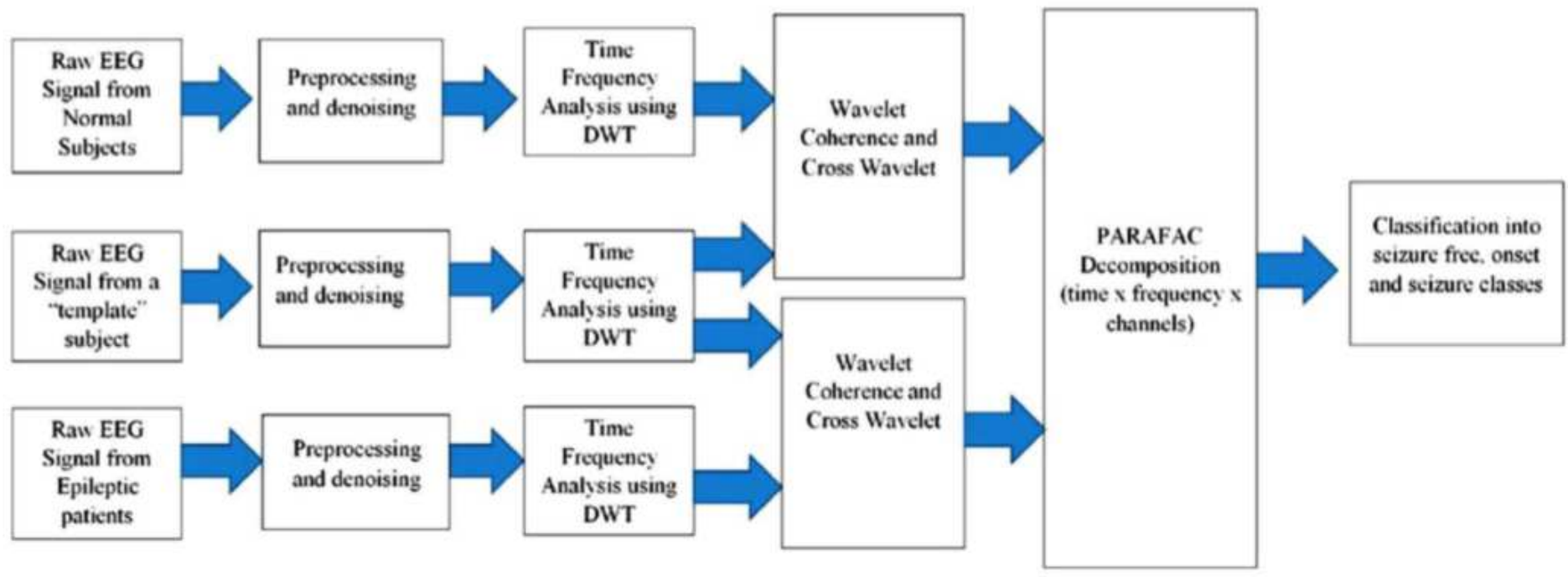

Figure 1

Block diagram of our proposed approach
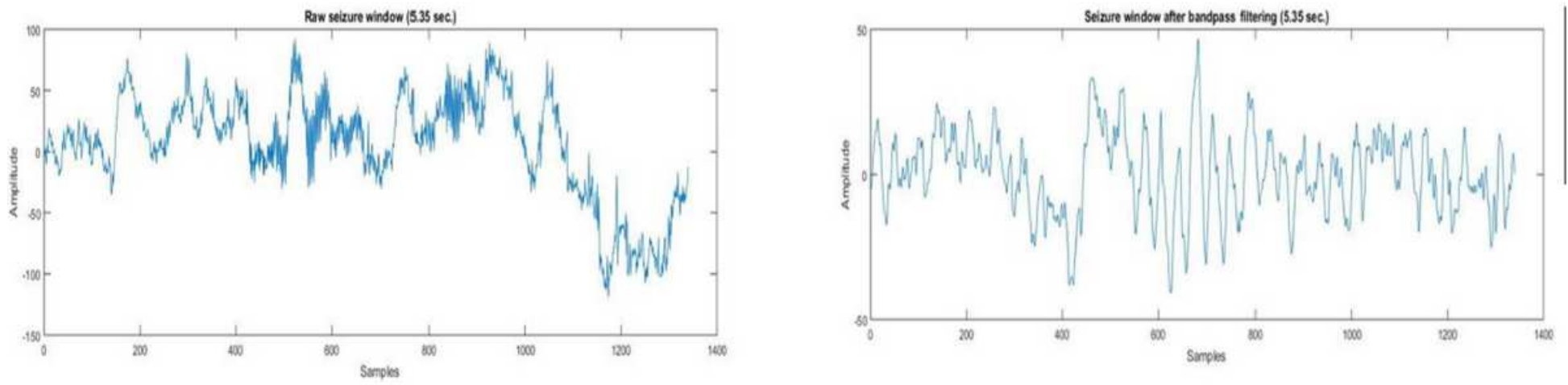

Figure 2

Pre-processing of seizure window (a) Raw seizure window (b) A seizure window after Bandpass filtering.
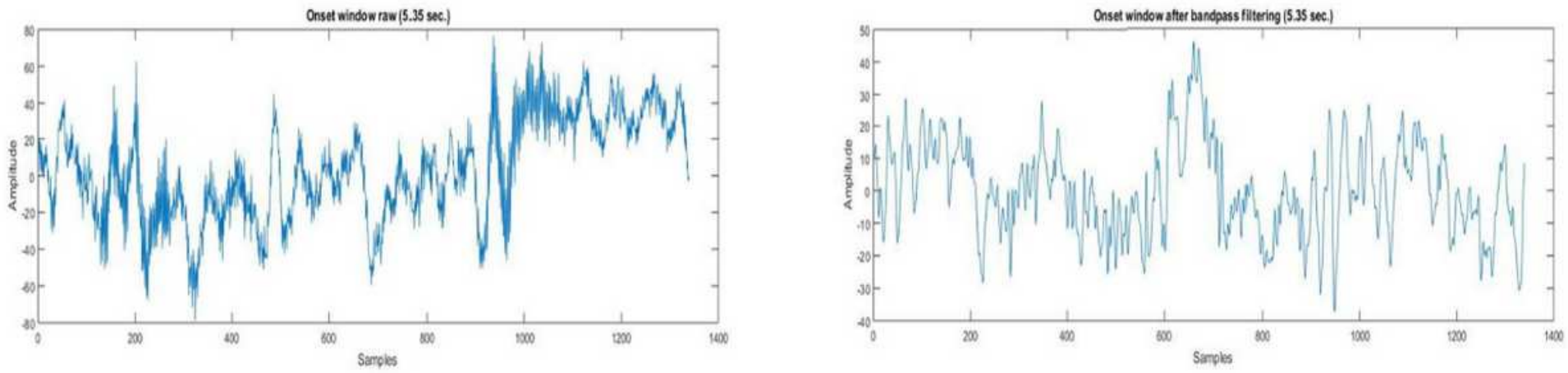

Figure 3 
Pre-processing of onset window (a) Raw onset window (b) An onset window after Bandpass filtering.
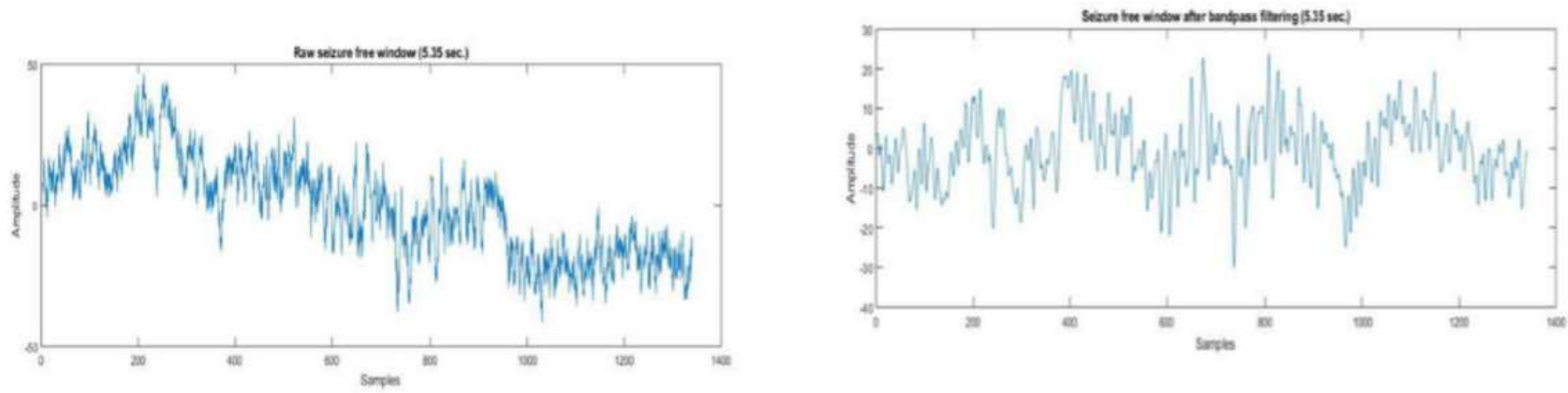

\section{Figure 4}

Pre-processing of seizure-free window (a) Raw seizure-free window (b) A seizure-free window after Bandpass filtering.

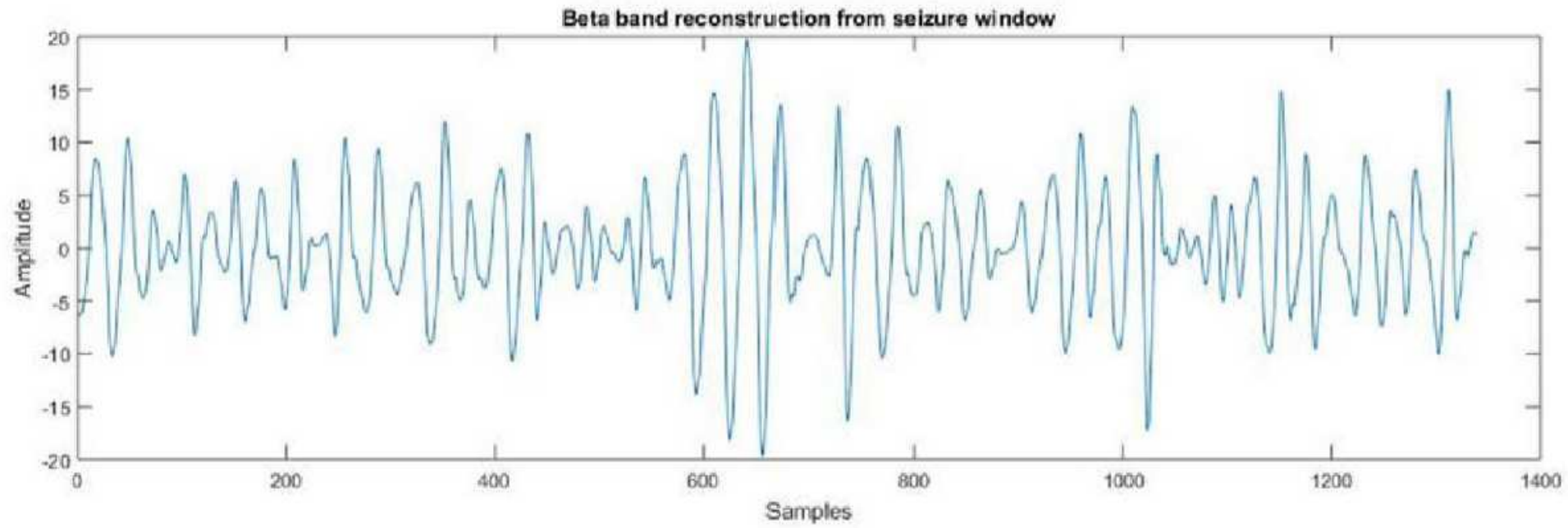

Figure 5

Beta band reconstruction from seizure window

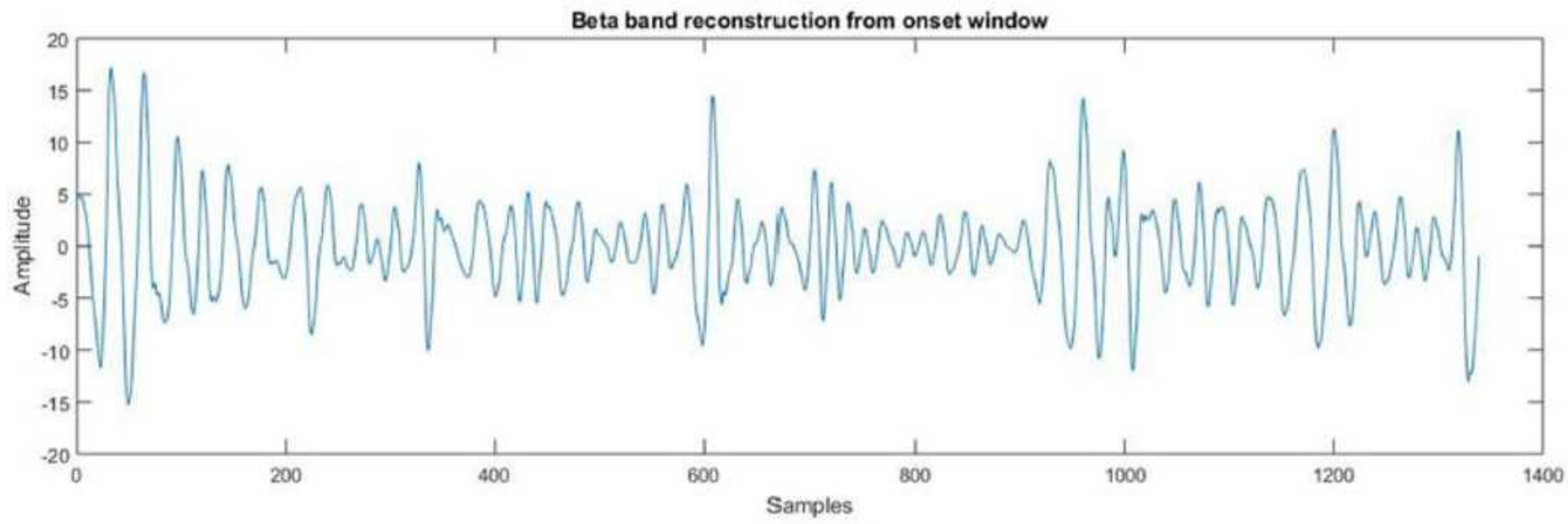


Figure 6

Beta band reconstruction from onset window

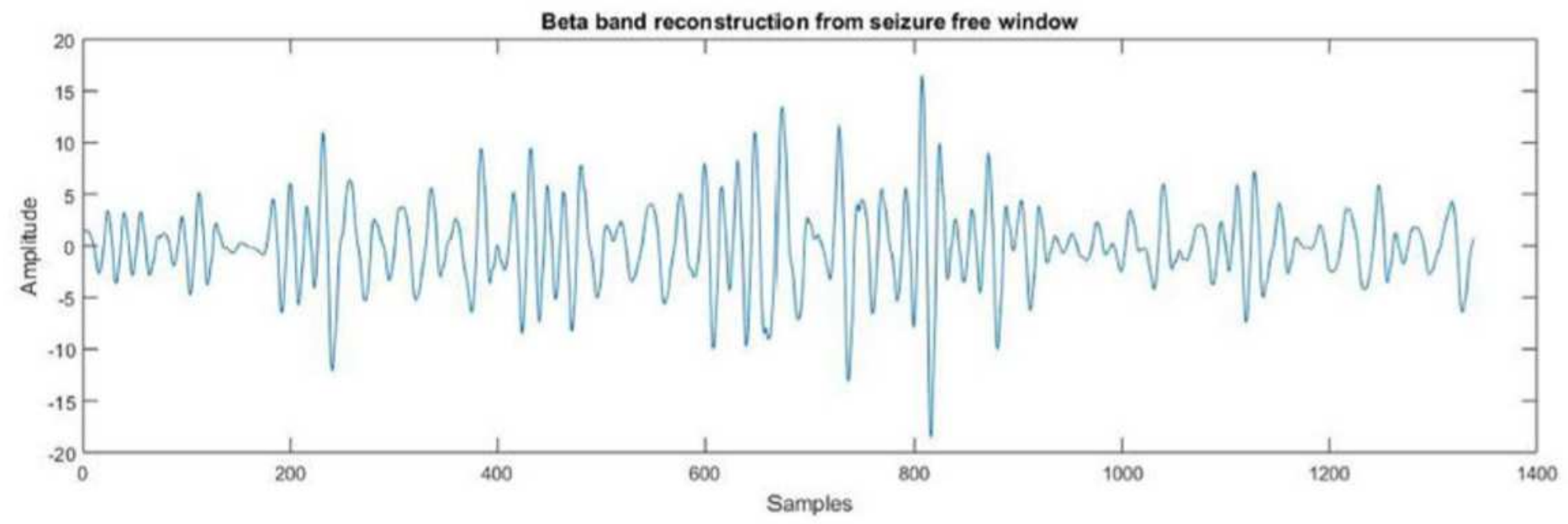

Figure 7

Beta band reconstruction from seizure-free window

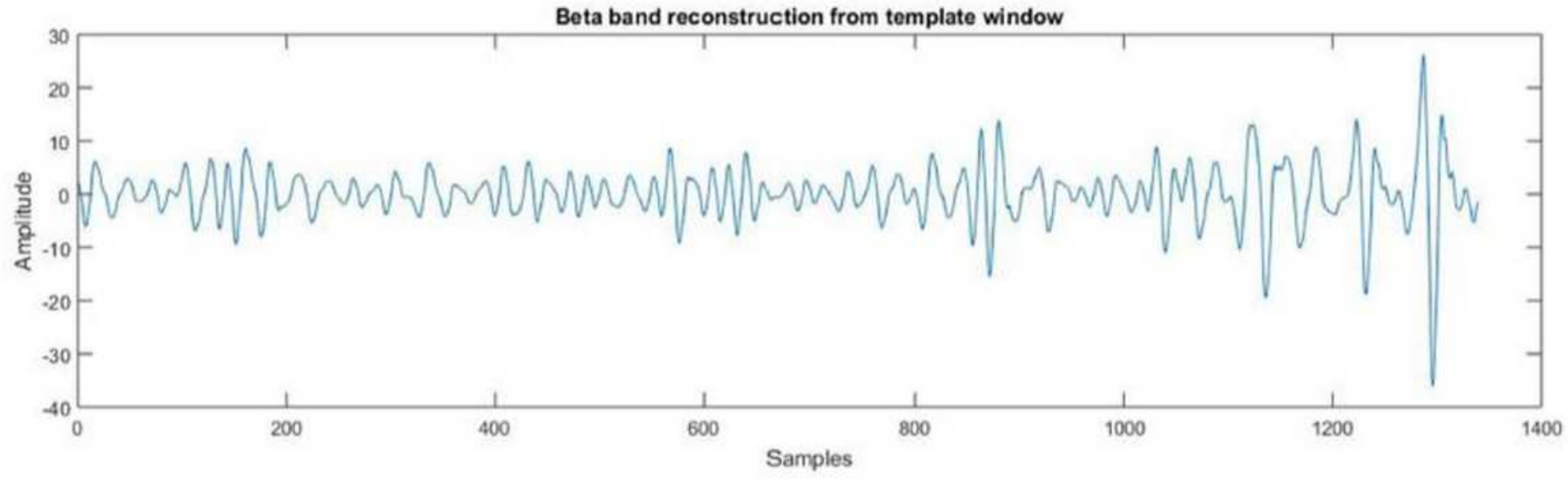

Figure 8

Beta band reconstruction from template window 

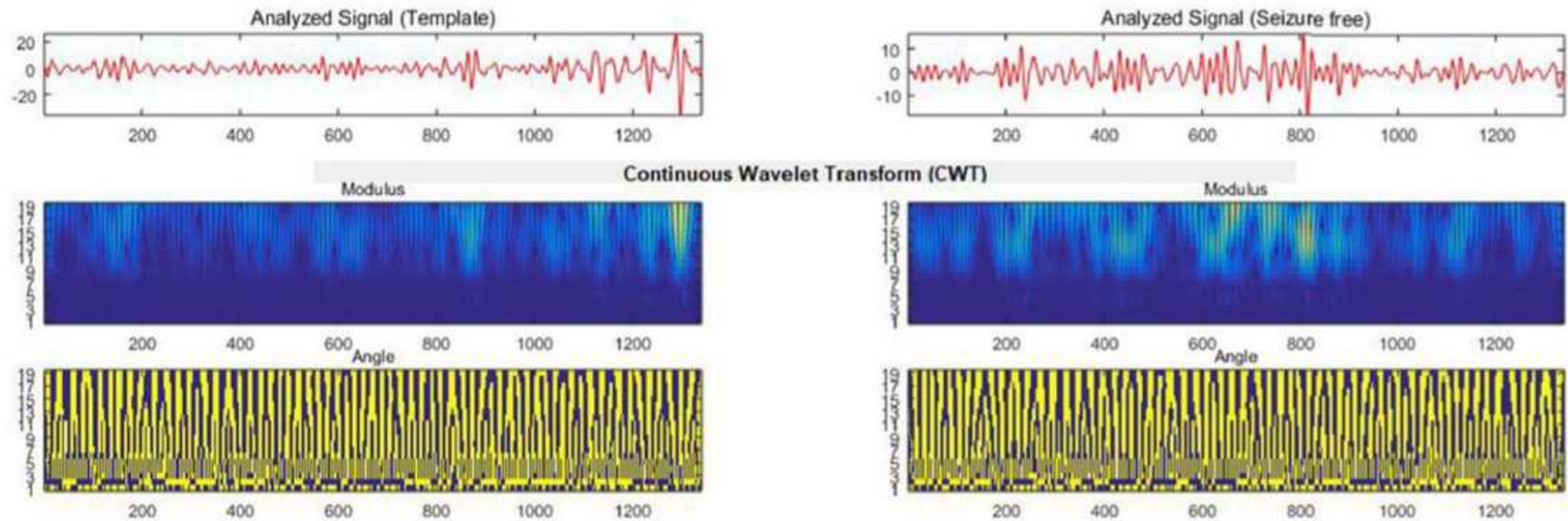

\section{Figure 9}

CWT for template and seizure-free
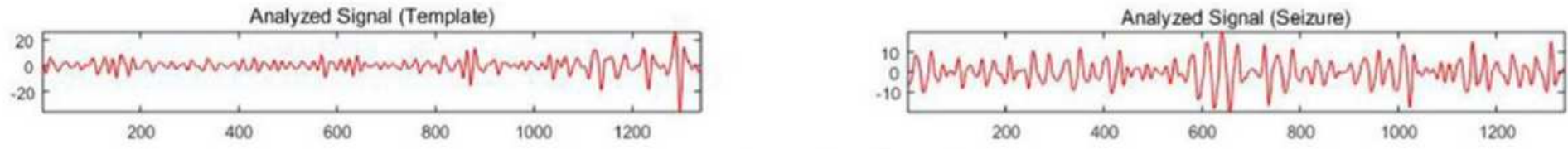

Continuous Wavelet Transform (CWT)

Modulus

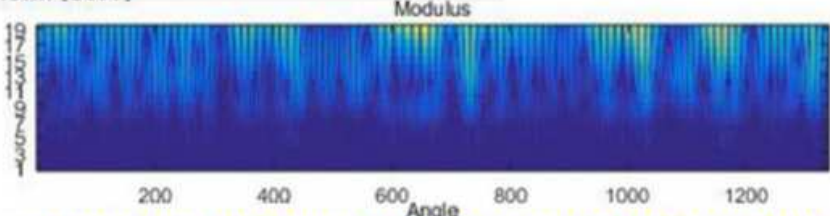

$\begin{array}{llllll}200 & 400 & 600 & 800 & 1000 & 1200\end{array}$

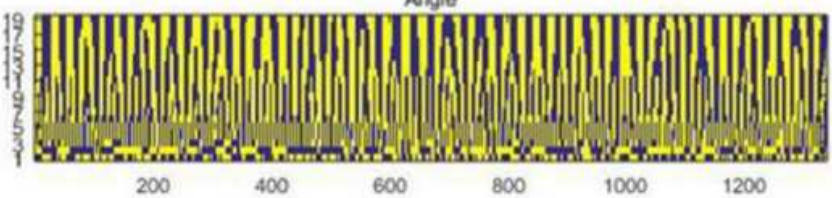

Figure 10

CWT for template and seizure 
Analyzed Signals (Template and Seizure free)
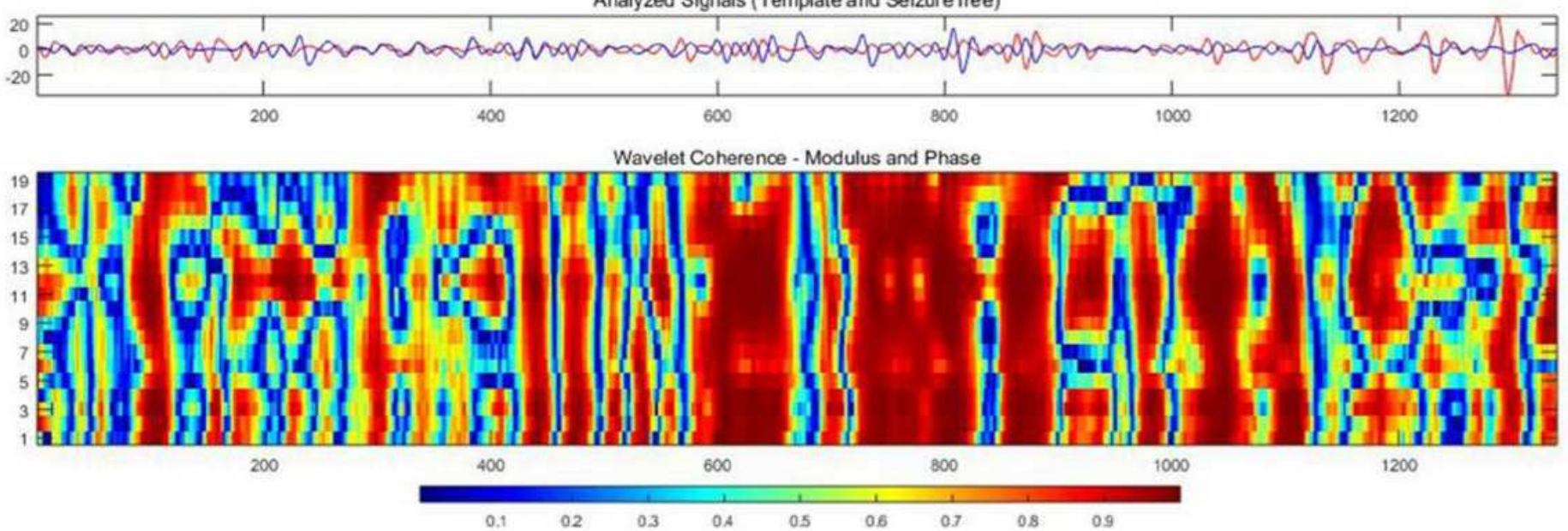

\section{Figure 11}

Wavelet Coherence for a template and a seizure-free window
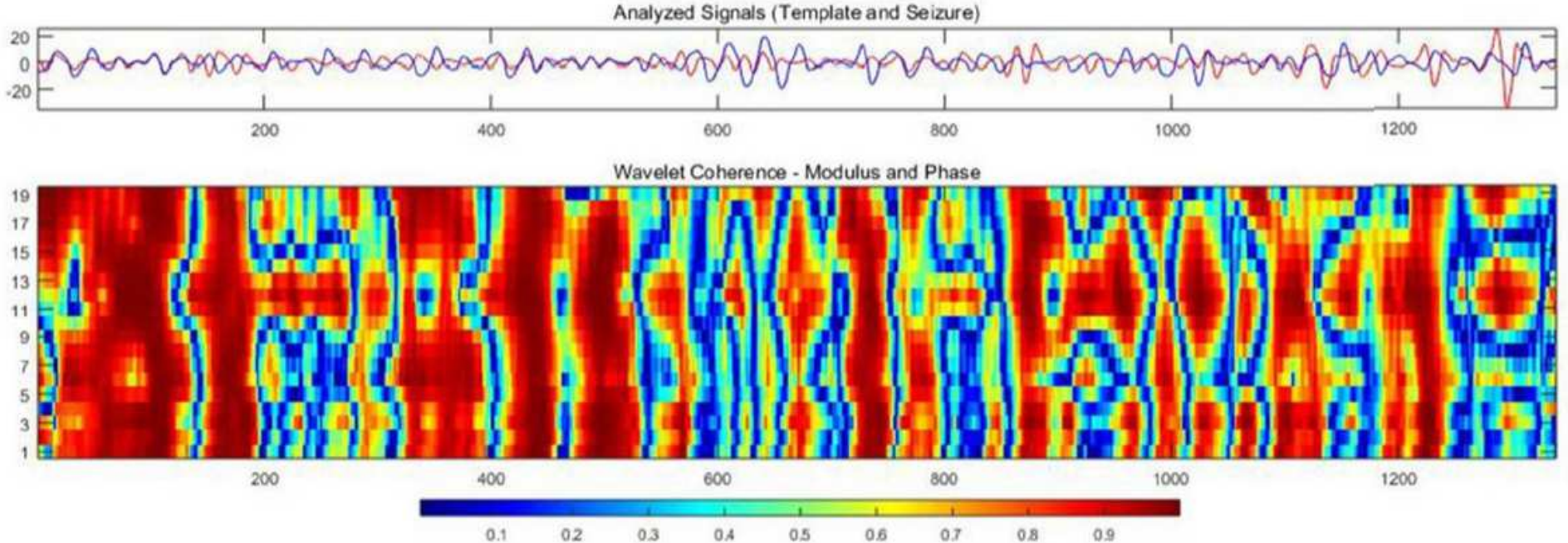

Figure 12

Wavelet Coherence for a template and a seizure window 
Analyzed Signals (Template and Seizure free)

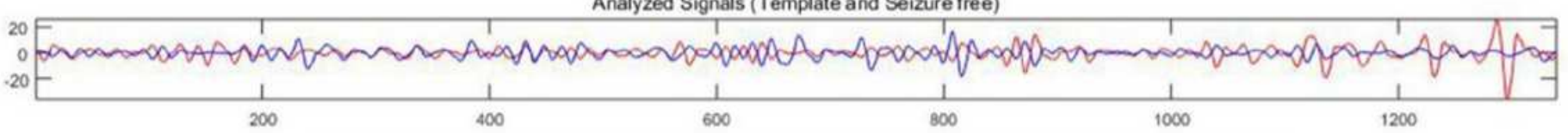

Wavelet Cross Spectrum (WCS)

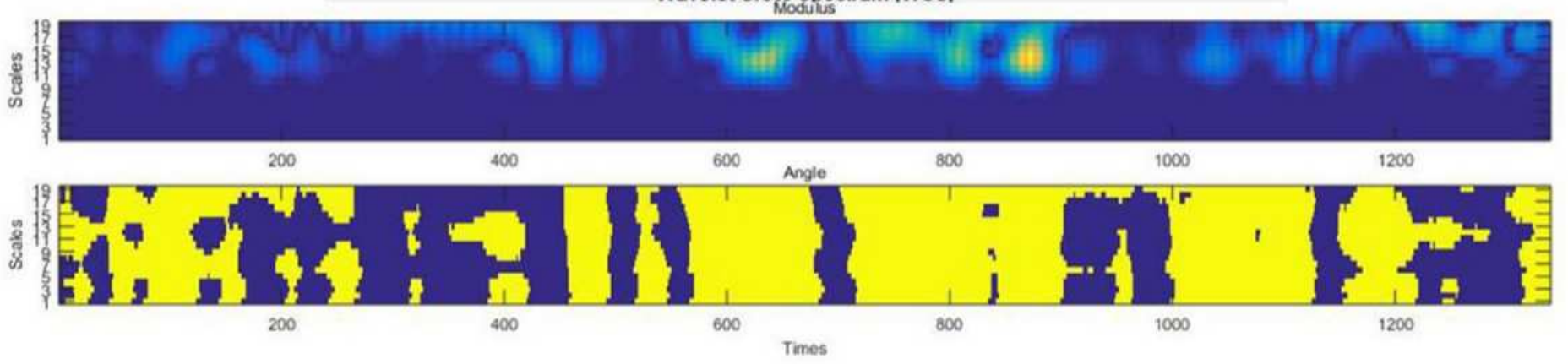

\section{Figure 13}

Wavelet Cross Spectrum for a template and a seizure-free window
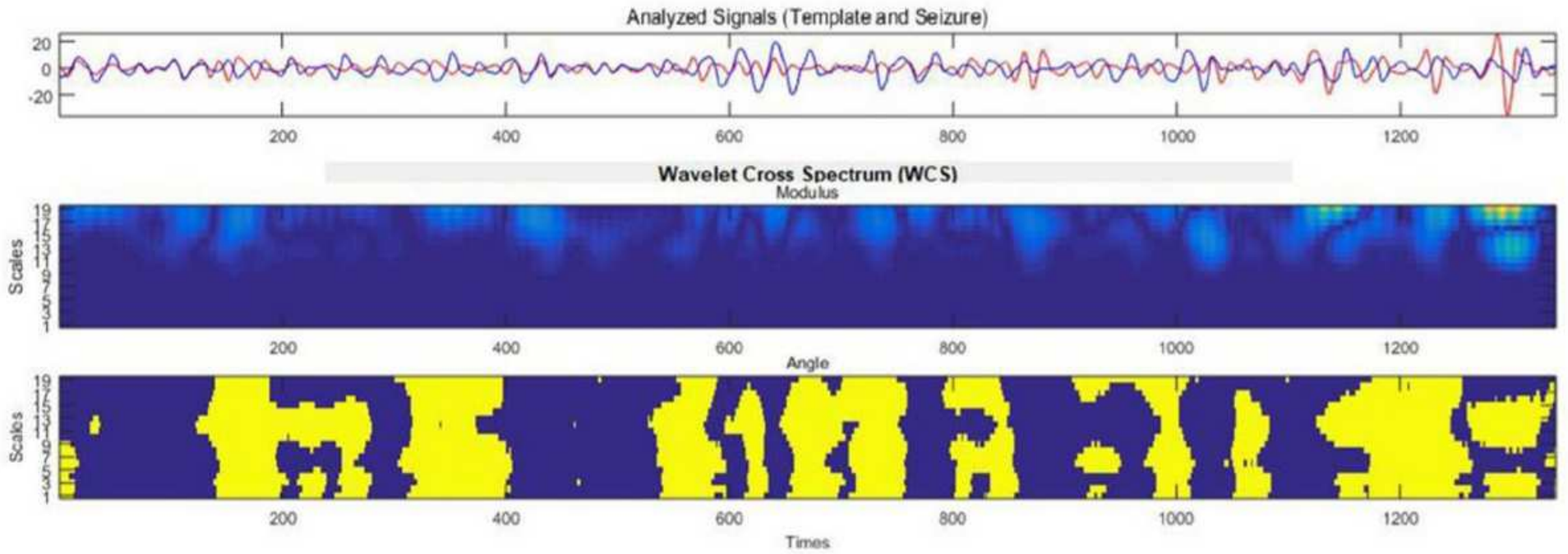

Figure 14

Wavelet Cross Spectrum for a template and a seizure window 


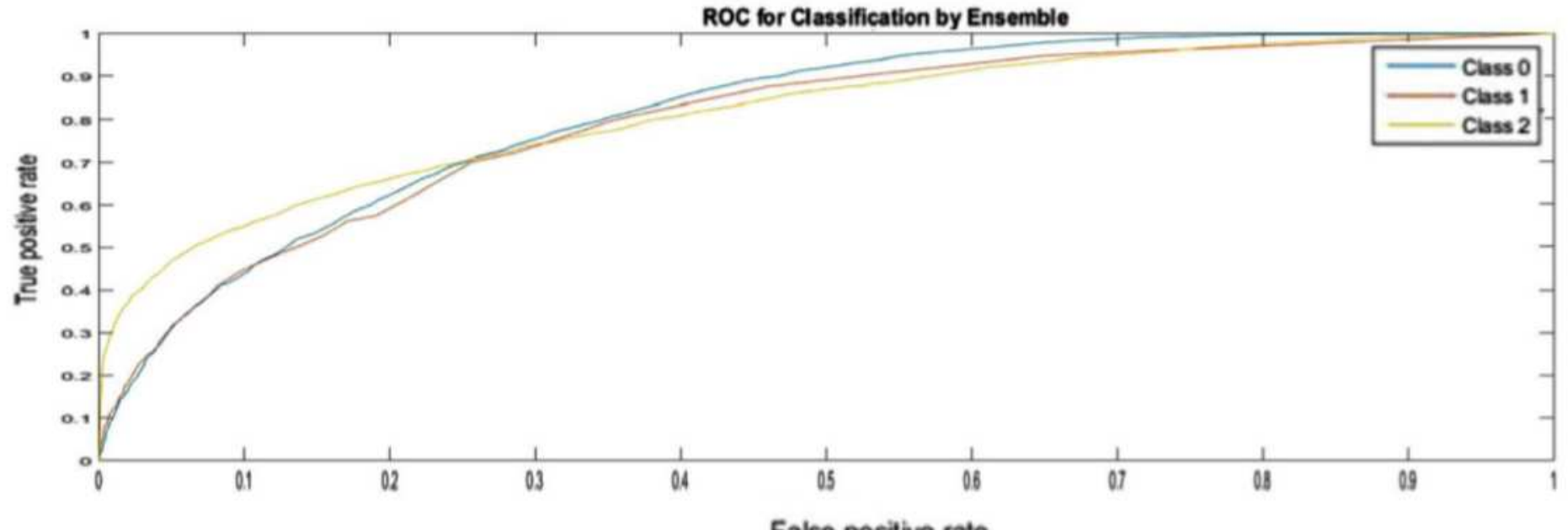

False positive rate

Figure 15

ROC curve for Classifier 3 (Ensemble Bagged Trees) for classification of the wavelet coherence feature

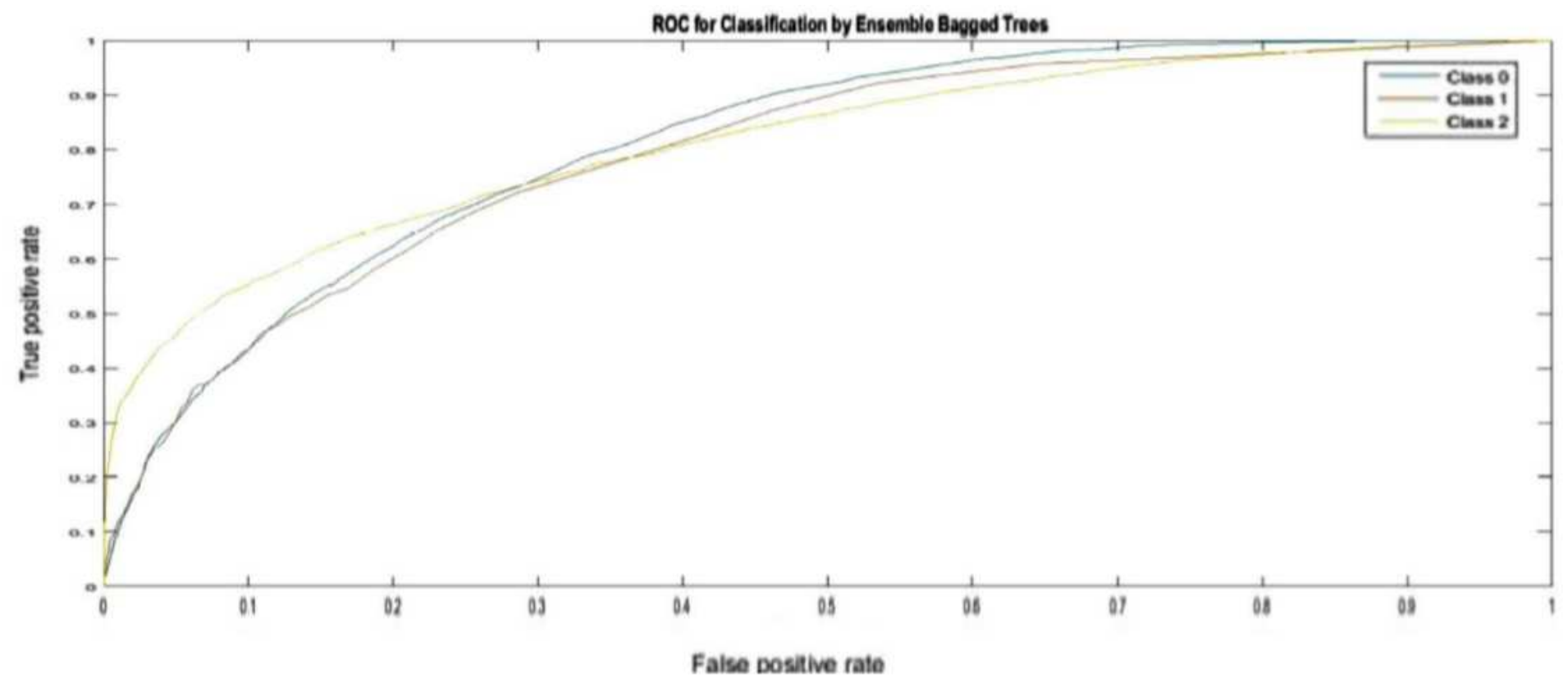

Figure 16

ROC curve for Classifier 3 (Ensemble Bagged Trees) for classification of the wavelet cross spectrum feature 